Roger Williams University

DOCS@RWU

2013

\title{
ILLUMINATING THE DARKEST GAMMA-RAY BURSTS WITH RADIO OBSERVATIONS
}

\author{
B.A. Zauderer \\ E. Berger \\ R. Margutti \\ A.J. Levan \\ F. Olivares E.
}

See next page for additional authors

Follow this and additional works at: https://docs.rwu.edu/fcas_fp

Part of the Astrophysics and Astronomy Commons

\section{Recommended Citation}

Zauderer. B.A. ...... A.C. Updike, et al. "Illuminating the Darkest Gamma-Ray Bursts with Radio Observations." Astrophysical Journal 767, (2013): 161.

This Article is brought to you for free and open access by the Arts and Sciences at DOCS@RWU. It has been accepted for inclusion in Arts \& Sciences Faculty Publications by an authorized administrator of DOCS@RWU. For more information, please contact mwu@rwu.edu. 


\section{Authors}

B.A. Zauderer, E. Berger, R. Margutti, A.J. Levan, F. Olivares E., D.A. Perley, W. Fong, A. Horesh, Adria Updike, J. Greiner, N.R. Tanvir, T. Laskar, R. Chornock, A.M. Soderberg, K.M. Menten, E. Nakar, J. Carpenter, P. Chandra, A.J. Castro-Tirado, M. Bremer, J. Gorosabel, S. Guziy, D. P'erez-Ram'ırez, and J.M. Winters 


\title{
ILLUMINATING THE DARKEST GAMMA-RAY BURSTS WITH RADIO OBSERVATIONS
}

\author{
B. A. Zauderer ${ }^{1}$, E. Berger ${ }^{1}$, R. Margutti ${ }^{1}$, A. J. Levan ${ }^{2}$, F. Olivares E. $^{3}$, D. A. Perley ${ }^{4,16}$, W. Fong $^{1}$, A. Horesh $^{4}$,

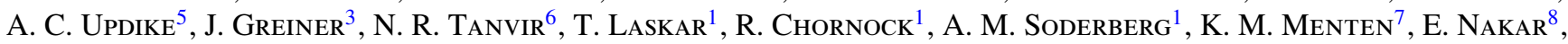 \\ J. Carpenter ${ }^{4}$, P. Chandra ${ }^{9}$, A. J. Castro-Tirado ${ }^{10}$, M. Bremer ${ }^{11}$, J. Gorosabel ${ }^{10,12,13}{ }^{\text {, S. GuziY }}{ }^{14}$, \\ D. PÉREZ-RAMíreZ ${ }^{15}$, AND J. M. WinTERS ${ }^{11}$ \\ ${ }^{1}$ Department of Astronomy, Harvard University, Cambridge, MA 02138, USA \\ ${ }^{2}$ Department of Physics, University of Warwick, Coventry CV4 7AL, UK \\ ${ }^{3}$ Max-Planck-Institut für extraterrestrische Physik, Giessenbachstraße, D-85748 Garching, Germany \\ ${ }^{4}$ Division of Physics, Mathematics and Astronomy, California Institute of Technology, Pasadena, CA 91225, USA \\ ${ }^{5}$ Department of Chemistry and Physics, Roger Williams University, Bristol, RI 02809, USA \\ ${ }^{6}$ Department of Physics and Astronomy, University of Leicester, Leicester LE1 7RH, UK \\ ${ }^{7}$ Max-Planck-Institut für Radioastronomie, D-53121 Bonn, Germany \\ ${ }^{8}$ Department of Astrophysics, Sackler School of Physics and Astronomy, Tel Aviv University, 69978 Tel Aviv, Israel \\ ${ }^{9}$ National Centre for Radio Astrophysics, Tata Institute of Fundamental Research, Pune University Campus, Ganeshkhind, Pune 411007, India \\ ${ }^{10}$ Instituto de Astrofísica de Andalucía (IAA-CSIC), P.O. Box 03004, E-18080 Granada, Spain \\ ${ }^{11}$ Institut de Radioastronomie Millimétrique, 300 rue de la Piscine, F-38406 Saint Martin d'Hères, France \\ ${ }^{12}$ Unidad Asociada Grupo Ciencia Planetarias UPV/EHU-IAA/CSIC, Departamento de Física Aplicada I, E.T.S. Ingeniería, \\ Universidad del País Vasco UPV/EHU, Alameda de Urquijo s/n, E-48013 Bilbao, Spain \\ ${ }^{13}$ Ikerbasque, Basque Foundation for Science, Alameda de Urquijo 36-5, E-48008 Bilbao, Spain \\ ${ }_{15}^{14}$ Nikolaev National University, Nikolskaya 24, 54030 Nikolaev, Ukraine \\ ${ }^{15}$ Universidad de Jaén, Campus Las Lagunillas s/n, E-23007 Jaén, Spain \\ Received 2012 September 19; accepted 2013 March 10; published 2013 April 8
}

\begin{abstract}
We present X-ray, optical, near-infrared (IR), and radio observations of gamma-ray bursts (GRBs) 110709B and 111215A, as well as optical and near-IR observations of their host galaxies. The combination of X-ray detections and deep optical/near-IR limits establish both bursts as "dark." Sub-arcsecond positions enabled by radio detections lead to robust host galaxy associations, with optical detections that indicate $z \lesssim 4(110709 \mathrm{~B})$ and $z \approx 1.8-2.9$ (111215A). We therefore conclude that both bursts are dark due to substantial rest-frame extinction. Using the radio and X-ray data for each burst we find that GRB $110709 \mathrm{~B}$ requires $A_{V}^{\text {host }} \gtrsim 5.3$ mag and GRB $111215 \mathrm{~A}$ requires $A_{V}^{\text {host }} \gtrsim 8.5 \mathrm{mag}$ (assuming $z=2$ ). These are among the largest extinction values inferred for dark bursts to date. The two bursts also exhibit large neutral hydrogen column densities of $N_{\mathrm{H} \text {,int }} \gtrsim 10^{22} \mathrm{~cm}^{-2}(z=2)$ as inferred from their X-ray spectra, in agreement with the trend for dark GRBs. Moreover, the inferred values are in agreement with the Galactic $A_{V}-N_{\mathrm{H}}$ relation, unlike the bulk of the GRB population. Finally, we find that for both bursts the afterglow emission is best explained by a collimated outflow with a total beaming-corrected energy of $E_{\gamma}+E_{K} \approx(7-9) \times 10^{51} \mathrm{erg}(z=2)$ expanding into a wind medium with a high density, $\dot{M} \approx(6-20) \times 10^{-5} M_{\odot} \mathrm{yr}^{-1}$ $\left(n \approx 100-350 \mathrm{~cm}^{-3}\right.$ at $\approx 10^{17} \mathrm{~cm}$ ). While the energy release is typical of long GRBs, the inferred density may be indicative of larger mass-loss rates for GRB progenitors in dusty (and hence metal rich) environments. This study establishes the critical role of radio observations in demonstrating the origin and properties of dark GRBs. Observations with the JVLA and ALMA will provide a sample with sub-arcsecond positions and robust host associations that will help to shed light on obscured star formation and the role of metallicity in GRB progenitors.
\end{abstract}

Key words: dust, extinction - gamma-ray burst: general

Online-only material: color figures

\section{INTRODUCTION}

Long-duration gamma-ray bursts (GRBs) have been linked to the deaths of massive stars, and hence to star formation activity, through their association with star-forming galaxies (e.g., Djorgovski et al. 1998; Fruchter et al. 2006; Wainwright et al. 2007) and with Type Ic supernova explosions (e.g., Woosley \& Bloom 2006). Across a wide range of cosmic history a substantial fraction of the star formation activity ( $~ 70 \%$ at the peak of the star formation history, $z \sim 2-4$ ) is obscured by dust, with about $15 \%$ of the total star formation rate density occurring in ultra-luminous infrared galaxies (e.g., Bouwens et al. 2009; Reddy \& Steidel 2009; Murphy et al. 2011). As a result, we expect some GRBs to occur in dusty environments that will

\footnotetext{
${ }^{16}$ Hubble Fellow
}

diminish or completely extinguish their optical (and perhaps even near-IR) afterglow emission. Such events can be used as signposts for the locations and relative fraction of obscured star formation across a wide redshift range (e.g., Reichart \& Price 2002; Ramirez-Ruiz et al. 2002; Trentham et al. 2002). In addition, they can provide insight into the role of metallicity in GRB progenitors since dusty environments generally require substantial metallicity (see Roseboom et al. 2012).

These so-called optically-dark GRBs are indeed known to exist (e.g., Groot et al. 1998; Djorgovski et al. 2001; Fynbo et al. 2001; Piro et al. 2002), but the lack of an optical detection does not necessarily point to dust obscuration. Most prosaically, the lack of detected optical emission may be due to inefficient follow-up observations, or to intrinsically dim events (e.g., Berger et al. 2002). Another potential origin of dark bursts is a high redshift, with the optical emission suppressed by 
Ly $\alpha$ absorption at $\lambda_{\text {obs }} \lesssim 1216 \AA \times(1+z)$ (Haislip et al. 2006; Salvaterra et al. 2009; Tanvir et al. 2009; Cucchiara et al. 2011). Such events are clearly of great interest since a dropout above $\sim 1 \mu \mathrm{m}$ points to $z \gtrsim 7$, while events that are also dark in the near-IR can potentially arise at $z \gtrsim 18$ ( $K$-band dropout). Naturally, high redshift bursts will also lack host galaxy detections in the optical band. On the other hand, their afterglow emission redward of the Ly $\alpha$ break will follow the expected synchrotron spectrum $\left(F_{v} \propto v^{-\beta}\right)$ with $\beta \approx 0.5-1$ (Sari et al. 1998).

To determine whether a burst is genuinely dark, due to extinction or to a high redshift rather than to an inefficient search, it is a useful diagnostic to compare the observed limits or faint detection with the expected optical/near-IR brightness expected based on the brightness of the X-ray afterglow. This approach relies on the simple power law shape of the afterglow synchrotron emission (see Sari et al. 1998). One definition of dark bursts uses an optical to X-ray spectral index of $\beta_{\mathrm{OX}} \lesssim 0.5$ ( $\beta$ is the slope of the synchrotron spectrum in log space; see Jakobsson et al. 2004 for details), since this is the shallowest expected slope in the standard afterglow model. A variant of this condition uses knowledge of the X-ray spectral index $\left(\beta_{\mathrm{X}}\right)$, and defines bursts as dark if $\beta_{\mathrm{OX}}-\beta_{\mathrm{X}} \lesssim-0.5$ (van der Horst et al. 2009), since this is the shallowest expected relative slope. These definitions can reveal evidence for dust extinction even if an optical afterglow is detected.

This approach of utilizing spectral slopes has been used by several groups to identify and study dark bursts, although different samples and analysis methods yield dramatically different results for the dark burst fraction and the mean extinction relative to the GRB population at large. In the preSwift era, Jakobsson et al. (2004) examined 52 bursts with $\mathrm{X}$-ray afterglows and identified five to be dark with $\beta_{\mathrm{OX}}<0.50$. However, they found another five to be potentially dark, strongly cautioning that each burst must be modeled individually and the $\beta_{\text {OX }}$ cutoff should only be used as a first order diagnostic. The overall fraction of dark bursts in the Jakobsson pre-Swift sample is somewhere between $\sim 10 \%$ and $20 \%$. To avoid selection bias, Fynbo et al. (2009) defined an X-ray-selected sample of 146 Swift bursts and constrained the dark burst fraction to be larger $(25 \%-42 \%)$ than the $14 \%$ dark burst fraction determined if only GRBs with spectroscopy are considered. Melandri et al. (2012) studied a complete sample of 58 bright Swift bursts, of which 52 have known redshifts and found that the fraction of dark bursts is about $30 \%$, mainly due to extinction (nearly all the dark bursts in their sample have $z \lesssim 4$ ). Thus, the afterglow emission of $\sim 1 / 3-1 / 2$ of all GRBs are affected by dust, although in most cases the required extinction is modest, $A_{V}^{\text {host }} \approx 0.3$ mag (Kann et al. 2006, 2010; Schady et al. 2007; Perley et al. 2009; Greiner et al. 2011).

Several studies have quantified dust extinction in GRBs. Analyzing optical/IR data for $30 \mathrm{GRBs}$ pre-Swift, Kann et al. (2006) found a mean extinction of $A_{V}^{\text {host }} \approx 0.2 \mathrm{mag}$, indicating that most events are not dark. Schady et al. (2007) studied several bursts with Swift X-ray and UV/optical detections and found a mean extinction level of $A_{V}^{\text {host }} \approx 0.3 \mathrm{mag}$, and that bursts that are dark blueward of $V$-band are likely to have rest-frame extinction about an order of magnitude larger. Melandri et al. (2008) used rapid optical observations of 63 Swift bursts and found that about $50 \%$ showed evidence of mild extinction. A similar conclusion was reached by Cenko et al. (2009) based on rapid optical observations of 29 Swift bursts, with an $80 \%$ detection fraction, but with roughly half exhibiting suppression with respect to the X-ray emission. A follow-up study of the latter sample by Perley et al. (2009) aimed at identifying host galaxies in the optical (thereby ruling out a high redshift origin) and found that $\lesssim 7 \%$ of Swift bursts are located at $z \gtrsim 7$. The majority of the dark bursts in the Perley et al. (2009) sample instead require $A_{V}^{\text {host }} \gtrsim 1 \mathrm{mag}$, with a few cases reaching $\sim 2-6$ mag. There are only a few known cases with large extinction of $A_{V}^{\text {host }} \sim$ few mag (see Perley et al. 2013, with 23 Swift galaxies having $A_{V}>1$ ). A few notable examples of highly extinguished afterglows include GRB 070306 (Jaunsen et al. 2008), GRB 080607 (Prochaska et al. 2009), and GRB 090417B (Holland et al. 2010).

Concurrent studies of the neutral hydrogen column density distribution, inferred from the X-ray afterglow spectra, suggest that dark bursts exhibit systematically larger values of $N_{\mathrm{H} \text {,int }} \gtrsim$ $10^{22} \mathrm{~cm}^{-2}$ compared with bursts with little or no extinction, which have a median of $N_{\mathrm{H} \text {,int }} \approx 4 \times 10^{21} \mathrm{~cm}^{-2}$ (Campana et al. 2012; Margutti et al. 2013). On the other hand, the measured extinction for GRBs is generally lower than expected based on the values of $N_{\mathrm{H} \text {,int }}$ and the Galactic $A_{V}-N_{\mathrm{H}}$ relation, previously attributed to dust destruction by the bright X-ray/UV emission (e.g., Galama \& Wijers 2001; Schady et al. 2007; Perley et al. 2009). Furthermore, Watson \& Jakobsson (2012) point out that the tendency for bursts with very high X-ray column to have dusty sight lines may introduce a bias that at least partially explains the observed trend of increasing $N_{\mathrm{H}, \mathrm{X}}$ with redshift. Above $z \sim 4$ there is typically less dust extinction, and so the high- $N_{\mathrm{H}, \mathrm{X}}$ bursts in this regime appear to require another explanation.

Finally, the host galaxies of at least some dark bursts appear to be redder, more luminous, more massive, and more metal rich than the hosts of optically-bright GRBs (Berger et al. 2007; Levesque et al. 2010; Perley et al. 2009, 2011a; Krühler et al. 2011), potentially indicating that the extinction is interstellar in origin rather than directly associated with the burst environment. One specific example is GRB 030115, not only one of the first examples of a heavily extinguished GRB afterglow, but residing in a host classified as an extremely red object (ERO; see Levan et al. 2006). More recently, Rossi et al. (2012) report seven additional potential ERO hosts. Some host galaxies of optically-bright bursts have been detected in the radio and (sub)millimeter ranges (e.g., Tanvir et al. 2004, indicating obscured star formation rates of $\sim 10^{2}-10^{3} M_{\odot} \mathrm{yr}^{-1}$ (Berger et al. 2003a). This suggests that the dust distribution within the host galaxies is patchy and that not all GRBs in dusty host galaxies are necessarily dust-obscured (Berger et al. 2003a; Perley et al. 2009). Further work by Svensson et al. (2012) indicates that dark GRB host galaxies may be systematically redder and more massive than optically bright GRB host galaxies and have lower metallicity than submillimeter galaxies, leading to prior biases in GRB host galaxy population studies.

Here we present multi-wavelength observations that reveal two of the darkest known bursts to date, GRBs 110709B and 111215A. These events have such large rest-frame extinction that they lack any afterglow detection in the optical and near-IR bands (to $\lambda_{\text {obs }} \approx 2.2 \mu \mathrm{m}$ ) despite rapid follow-up. However, both events are detected in the radio, providing sub-arcsecond positions and secure associations with host galaxies detected in the optical. This allows us to rule out a high redshift origin, while the combination of radio and X-ray data place robust lower limits on the rest-frame extinction, with $A_{V}^{\text {host }} \gtrsim 5.3$ and $\gtrsim 8.5 \mathrm{mag}$ (for $z=2$ ), respectively. The radio and $\mathrm{X}$-ray data also allow us to determine the explosion properties 
and the circumburst densities. This study demonstrates that radio detections with the JVLA, and soon with the Atacama Large Millimeter/submillimeter Array (ALMA), provide a promising path to accurate localization of dark GRBs, robust estimates of the extinction in the absence of any optical/near-IR detections, and a comparative study of their explosion properties and parsecscale environments.

The plan of the paper is as follows. We describe the multiwavelength observations of GRBs 110709B and 111215A and their host galaxies in Section 2. These observations establish that both events are dark due to extinction. We model the radio and X-ray data to extract the minimum required extinction, as well as the explosion and circumburst properties in Section 3. In Section 4 we place the large inferred extinction values and the inferred burst properties within the framework of existing samples of optically-bright and dark bursts. We summarize the results and note key future directions in Section 5. Throughout the paper we report magnitudes in the $\mathrm{AB}$ system (unless otherwise noted) and use Galactic extinction values of $E(B-$ $V) \approx 0.044 \mathrm{mag}$ for GRB $110709 \mathrm{~B}$ and $E(B-V) \approx 0.057 \mathrm{mag}$ for GRB 111215A (Schlafly \& Finkbeiner 2011). We use the standard cosmological parameters: $H_{0}=71 \mathrm{~km} \mathrm{~s}^{-1} \mathrm{Mpc}^{-1}$, $\Omega_{\Lambda}=0.73$, and $\Omega_{\mathrm{M}}=0.27$.

\section{OBSERVATIONS}

\subsection{Discovery and Burst Properties}

\subsubsection{GRB $110709 B$}

GRB 110709B was discovered on 2011 July 9 at 21:32:39 UT (Cummings et al. 2011b) by the Swift Burst Alert Telescope (BAT; 15-150 keV; Barthelmy et al. 2005), and by KonusWIND (20 keV-5 MeV; Golenetskii et al. 2011). Swift X-ray Telescope (XRT; 0.3-10 keV; Burrows et al. 2005) observations commenced at $\delta t \approx 80 \mathrm{~s}$ (Cummings et al. 2011b), localizing the X-ray afterglow to R.A. $=10^{\mathrm{h}} 58^{\mathrm{m}} 37^{\mathrm{s}} .08$, decl. $=-23^{\circ} 27^{\prime} 17^{\prime \prime} .6$ (J2000), with an uncertainty of 1".4 (90\% containment, UVOTenhanced; Beardmore et al. 2011a). Swift UV/Optical Telescope (UVOT; Roming et al. 2005) observations began at $\delta t \approx 90 \mathrm{~s}$, but no afterglow candidate was identified in $3107 \mathrm{~s}$ to a $3 \sigma$ limit of $23.0 \mathrm{mag}$ in the white filter (Holland \& Cummings 2011). A large flare was detected with the BAT, XRT, and Konus-WIND at $\delta t \approx 11$ minutes with an intensity comparable to the main event (Barthelmy et al. 2011b).

Zhang et al. (2012) presents a detailed analysis of the prompt emission. The initial trigger had a duration and fluence in the BAT $15-150 \mathrm{keV}$ band of $T_{90} \approx 56 \mathrm{~s}$ and $F_{\gamma}=$

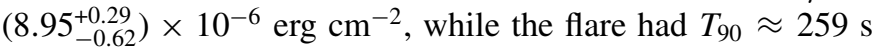
and $F_{\gamma}=\left(1.34_{-0.07}^{+0.05}\right) \times 10^{-5} \mathrm{erg} \mathrm{cm}^{-2}$ (Zhang et al. 2012). No emission was detected in the BAT band at $\delta t \approx 180-485 \mathrm{~s}$. Combining both events, the resulting duration is $T_{90} \approx 846 \pm 6 \mathrm{~s}$ (Cummings et al. 2011a). The initial trigger had a fluence in the Konus-WIND 20-5000 keV band of $(2.6 \pm 0.2) \times 10^{-5} \mathrm{erg} \mathrm{cm}^{-2}$. Zhang et al. (2012) calculate the total fluence in the Swift/XRT band to be $4.07 \pm 0.56 \times 10^{-6} \mathrm{erg} \mathrm{cm}^{-2}$.

\subsubsection{GRB 111215A}

GRB 111215A was discovered on 2011 December 15 at 14:04:08 UT by the Swift/BAT (Oates et al. 2011). Swift/XRT observations began at $\delta t \approx 409 \mathrm{~s}$ and localized the X-ray afterglow to R.A. $=23^{\mathrm{h}} 18^{\mathrm{m}} 13^{\mathrm{s}} .29$, decl. $=+32^{\circ} 29^{\prime} 38^{\prime \prime} .4$, with an uncertainty of 1 ". 4 (90\% containment, UVOT-enhanced;
Beardmore et al. 2011b). No afterglow candidate was identified by the UVOT in $645 \mathrm{~s}$ to a $3 \sigma$ limit of $\gtrsim 22.0 \mathrm{mag}$ in the white filter (Oates 2011; Oates et al. 2012). The duration and fluence are $T_{90} \approx 796 \mathrm{~s}$ and $F_{\gamma}=(4.5 \pm 0.5) \times 10^{-6} \mathrm{erg} \mathrm{cm}^{-2}$ (15-150 keV; Barthelmy et al. 2011a).

\subsection{X-Ray Observations}

\subsubsection{GRB 110709B}

We analyzed the XRT data using the HEASOFT package (v6.11) and corresponding calibration files. We utilized standard filtering and screening criteria, and generated a count-rate light curve following the prescriptions by Margutti et al. (2010). The data were re-binned with the requirement of a minimum signalto-noise ratio $(\mathrm{S} / \mathrm{N})$ of 4 in each temporal bin.

The XRT data exhibit significant spectral evolution during the early bright phase ( $\delta t \lesssim 1 \mathrm{ks}$ ), with spectral hardening when the burst re-brightened at $\sim 11$ minutes. Due to this variation, we performed a time-resolved spectral analysis, accumulating signal over time intervals defined to contain a minimum of about 1000 photons. Spectral fitting was done with Xspec (v12.6; Arnaud 1996), assuming a photoelectrically absorbed power law model and a Galactic neutral hydrogen column density of $N_{\mathrm{H}, \mathrm{MW}}=5.6 \times 10^{20} \mathrm{~cm}^{-2}$ (Kalberla et al. 2005). We extracted a spectrum in a time interval when no spectral evolution was apparent (15-150 ks) and used this to estimate the contribution of additional absorption. We find that the data are best modeled by an absorbed power-law ${ }^{17}$ model with a spectral photon index of $\Gamma=2.3 \pm 0.1$ and excess absorption of $N_{\mathrm{H} \text {,int }}=(1.4 \pm 0.2) \times 10^{21} \mathrm{~cm}^{-2}$ at $z=0(90 \%$ c.l., C-stat $=$ 559 for 604 degrees of freedom). The excess absorption was used as a fixed parameter in the time-resolved spectral analysis, allowing us to derive a count-to-flux conversion factor for each spectrum. The resulting unabsorbed $0.3-10 \mathrm{keV}$ flux light curve properly accounts for spectral evolution of the source. We note that for the purpose of display in Figure 1 we binned the XRT data by orbit at $\delta t \lesssim 2$ days and by multiple orbits thereafter.

We also observed GRB $110709 \mathrm{~B}$ for $15 \mathrm{ks}$ with the Advanced CCD Imaging Spectrometer (ACIS-S) on-board the Chandra $\mathrm{X}$-ray Observatory on 2011 July $23.60 \mathrm{UT}(\delta t \approx 13.7$ days $)$. The afterglow is detected with a count rate of $(4.1 \pm 0.5) \times 10^{-3} \mathrm{~s}^{-1}$ (0.5-8 keV), corresponding to an unabsorbed $0.3-10 \mathrm{keV}$ flux of $(6.0 \pm 0.7) \times 10^{-14} \mathrm{erg} \mathrm{s}^{-1} \mathrm{~cm}^{-2}$ using the XRT spectral parameters. Relative astrometry with the Chandra position will be discussed in Section 2.6. A second, $9 \mathrm{ks}$ Chandra/ACIS-S observation was performed on 2011 October $31.83 \mathrm{UT}(\delta t \approx 113.4$ days $)$. The $\mathrm{X}$-ray afterglow is not detected to a $3 \sigma$ limit of $\lesssim 9 \times 10^{-15}$ erg s $\mathrm{sm}^{-1}$, well above the extrapolation of the early light curve to this epoch (Figure 1).

At $\delta t \gtrsim 12$ days the XRT light curve flattens to a level of about $10^{-13} \mathrm{erg} \mathrm{s}^{-1} \mathrm{~cm}^{-2}$. The Chandra observations reveal that this is due to a contaminating source located about 3".8 from the GRB position, well within the point spread function (PSF) of XRT. We subtract the flux of this source, $(6.0 \pm 0.7) \times 10^{-14} \mathrm{erg} \mathrm{s}^{-1} \mathrm{~cm}^{-2}$, from the XRT light curve of GRB 110709B in Figure $1 .{ }^{18}$

\subsubsection{GRB 111215A}

We analyzed the XRT data for GRB 111215A in the same manner described above. At $\delta t \lesssim 2$ ks the light curve exhibits

\footnotetext{
17 tbabs $\times$ ztbabs $\times$ pow.

18 We note that the flux from the contaminating source is constant between the two epochs of Chandra, and rules out any origin as a lensed counterpart of the GRB, which may have caused the two $\gamma$-ray triggers.
} 


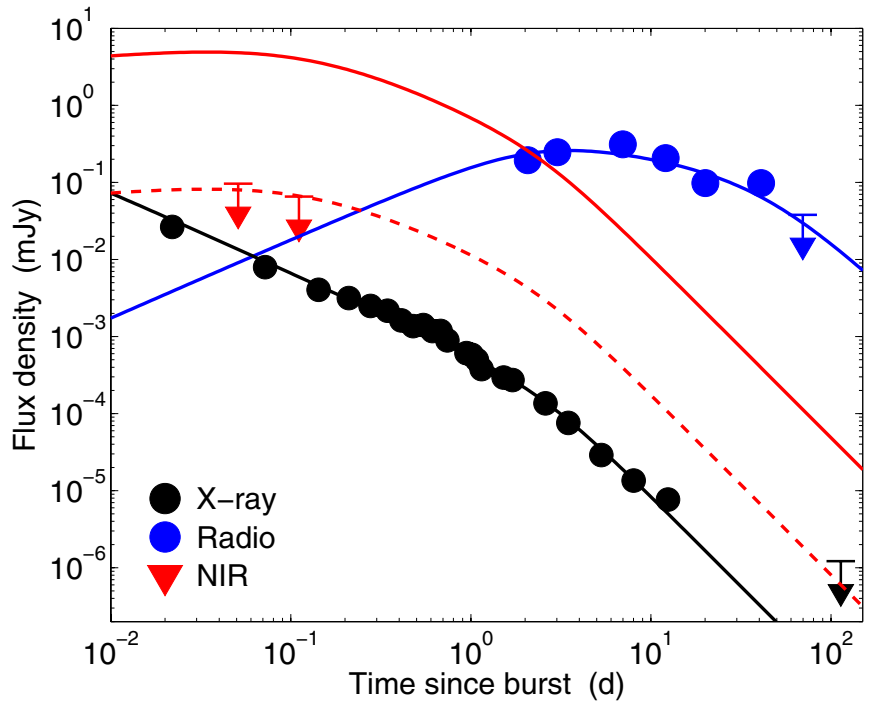

Figure 1. X-ray (black) and radio (blue) light curves of GRB 110709B, with near-IR limits (red triangles). For the purpose of display, the XRT data are binned by orbit at $\delta t \lesssim 2$ days and by multiple orbits thereafter. The Chandra $3 \sigma$ upper limit at $\delta t \approx 113$ days is indicated with a black arrow. Afterglow model fits in each band are shown with solid lines. To satisfy the near-IR upper limits we require $A_{V} \gtrsim 3.4-10.5 \mathrm{mag}$ (for $z=4$ to 1 ; dashed red line). The derived afterglow parameters for GRB 110709B are listed in Table 4.

(A color version of this figure is available in the online journal.)

flares with a hard-to-soft spectral evolution. To obtain a reliable estimate of the intrinsic neutral hydrogen absorption in addition to the Galactic value $\left(N_{\mathrm{H}, \mathrm{MW}}=5.5 \times 10^{20} \mathrm{~cm}^{-2}\right.$; Kalberla et al. 2005), we extracted a spectrum collecting the photon count data in the time interval $5-2000 \mathrm{ks}$, when no spectral evolution is apparent. The data are best modeled by an absorbed power-law with $\Gamma=2.2 \pm 0.1$ and $N_{\mathrm{H}, \text { int }}=(3.1 \pm 0.4) \times 10^{21} \mathrm{~cm}^{-2}$ at $z=0$ (90\% c.l., C-stat $=499.13$ for 512 degrees of freedom). The resulting unabsorbed $0.3-10 \mathrm{keV}$ flux light curve is shown in Figure 2.

\subsection{Optical/Near-IR Afterglow Limits}

\subsubsection{GRB 110709B}

We observed GRB 110709B with the Gemini Multi-Object Spectrograph (GMOS; Hook et al. 2004) on the Gemini-South $8 \mathrm{~m}$ telescope in $r$-band on 2011 July 10.03 and $13.96 \mathrm{UT}$ $(\delta t \approx 3.2 \mathrm{hr}$ and $\approx 4.1$ days). We analyzed the data with the gemini package in IRAF, but did not detect any sources within the XRT error circle to $3 \sigma$ limits of $\gtrsim 23.8 \mathrm{mag}$ ( $\delta t \approx 3.2 \mathrm{hr}$ ) and $\gtrsim 25.1 \mathrm{mag}$ ( $\delta t \approx 4.1$ days). A comparison of the early $r$-band limit $\left(F_{v, \text { opt }} \lesssim 1.1 \mu \mathrm{Jy}\right)$ to the X-ray flux density at the same time $\left(F_{\nu, \mathrm{X}} \approx 4.0 \mu \mathrm{Jy}\right)$ indicates an optical to X-ray spectral index $\beta_{\mathrm{OX}} \lesssim-0.2$. This is substantially flatter than the minimum expected spectral index of $\beta_{\mathrm{OX}}=0.5$, indicating that GRB $110709 \mathrm{~B}$ is a dark burst; for $\beta_{\mathrm{OX}}=0.5$ the expected $r$-band brightness is $\approx 19 \mathrm{mag}$. Similarly, relative to the $\mathrm{X}$-ray spectral index we find $\beta_{\mathrm{OX}}-\beta_{\mathrm{X}} \lesssim-1.5$, clearly satisfying the dark burst condition $\beta_{\mathrm{OX}}-\beta_{\mathrm{X}} \lesssim-0.5$.

We also observed the burst with the Gamma-Ray Burst Optical/Near-Infrared Detector (GROND; Greiner et al. 2007, 2008) mounted on the Max Planck Gesellschaft/European Southern Observatory $2.2 \mathrm{~m}$ telescope at La Silla Observatory starting on 2011 July $9.94 \mathrm{UT}(\delta t \approx 1 \mathrm{hr})$ simultaneously in $g^{\prime} r^{\prime} i^{\prime} z^{\prime} J H K_{\mathrm{s}}$, with an average seeing of $1^{\prime \prime} .5$ and an average airmass of 1.3 . We analyzed the data using standard pyraf/IRAF

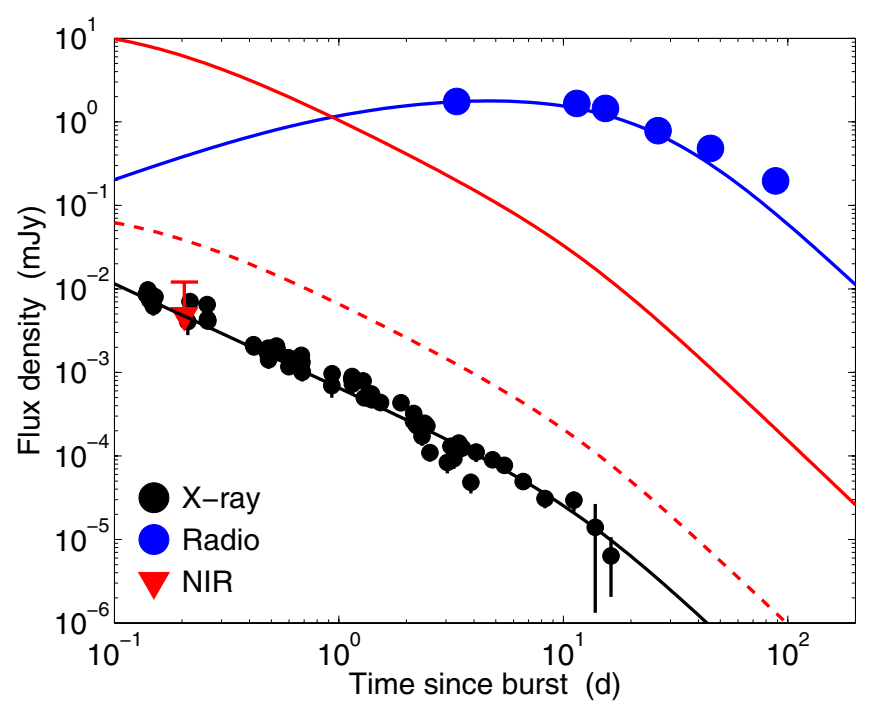

Figure 2. X-ray (black) and radio (blue) light curves of GRB 111215A, with near-IR limits (red triangle). Afterglow model fits in each band are shown with solid lines. To explain the near-IR upper limit we require $A_{V} \gtrsim 8.5 \mathrm{mag}$ (dashed line, assuming $z=2$ ). The derived afterglow parameters are listed in Table 5 . (A color version of this figure is available in the online journal.)

tasks, following the procedures described in Krühler et al. (2008). We calibrated the optical channels with zero-points computed during photometric conditions, and the near-IR channels using stars in the Two Micron All Sky Survey (2MASS) catalog. Calibration uncertainties vary in the range 0.02-0.12 mag for $J H K_{\mathrm{s}}$, and we add these systematic errors with the statistical errors in quadrature. We do not detect any source in the individual or stacked images within the XRT error circle. The upper limits are listed in Table 1, derived by forcing the photometry at the position of the radio counterpart (Section 2.4). A comparison of the $K_{\mathrm{s}}$ band limit at $\delta t \approx 2.7 \mathrm{hr}\left(F_{\nu, \mathrm{NIR}} \lesssim 69 \mu \mathrm{Jy}\right)$ to the X-ray flux density at the same time indicates a near-IR to X-ray slope of $\lesssim 0.35$, indicating that GRB $110709 \mathrm{~B}$ is a dark burst in the near-IR as well.

The absence of optical/near-IR emission can be due to dust extinction, or alternatively to a high redshift, $z \gtrsim 18$ based on the $K_{\mathrm{s}}$-band non-detection. We rule out a high redshift origin in Section 2.6.

\subsubsection{GRB $111215 A$}

Several optical afterglow searches on timescales of $\delta t \approx$ 7 minutes to $6.8 \mathrm{hr}$ led to non-detections of an afterglow with limits of $\gtrsim 17.5-22.8 \mathrm{mag}$ in various filters (e.g., Xin 2011; Xu et al. 2011; Usui et al. 2011; Pandey et al. 2011; Gorbovskoy et al. 2011; Rumyantsev et al. 2011). We observed the position of GRB 111215A with the Observatorio de Sierra Nevada (OSN) $1.5 \mathrm{~m}$ telescope beginning 2011 December $15.75 \mathrm{UT}(\delta \mathrm{t} \sim 4 \mathrm{hr})$ and obtained an early deep $i$-band limit of $m_{i} \gtrsim 21.7 \mathrm{mag}$. The deepest optical limit is $m_{R} \gtrsim 22.8 \mathrm{mag}$ at a mid-time of $\delta t \approx 37$ minutes (Xu et al. 2011), corresponding to a flux density of $F_{v \text {,opt }} \lesssim 2.3 \mu \mathrm{Jy}$. Near-IR observations also led to non-detections (D’Avanzo et al. 2011; Tanvir et al. 2011), with the deepest limit being $m_{K} \gtrsim 19.3 \mathrm{mag}(\mathrm{Vega})$ at $\delta t \approx 0.20$ days (Tanvir et al. 2011), corresponding to $F_{v, \mathrm{NIR}} \lesssim 12 \mu \mathrm{Jy}$.

A comparison of the deepest optical limit to the $\mathrm{X}$-ray flux density at the same time $\left(F_{v, \mathrm{X}} \approx 54 \mu \mathrm{Jy}\right)$ indicates $\beta_{\mathrm{OX}} \lesssim-0.5$. Similarly, a comparison of the deepest near-IR limit to the $\mathrm{X}$-ray flux density at the same time $\left(F_{v, \mathrm{X}} \approx 4.2 \mu \mathrm{Jy}\right)$ indicates 
Table 1

GROND Observations of GRB 110709B

\begin{tabular}{|c|c|c|c|c|c|c|c|c|c|c|}
\hline \multirow[t]{2}{*}{ UT Date } & \multirow{2}{*}{$\begin{array}{c}\delta t \\
\text { (days) }\end{array}$} & \multirow{2}{*}{$\begin{array}{l}\text { Integration } \\
\text { (s) }\end{array}$} & \multicolumn{4}{|c|}{ Upper Limits } & \multirow{2}{*}{$\begin{array}{l}\text { Integration } \\
\text { (s) }\end{array}$} & \multicolumn{3}{|c|}{ Upper Limits } \\
\hline & & & $g^{\prime}$ & $r^{\prime}$ & $i^{\prime}$ & $z^{\prime}$ & & $J$ & $H$ & $K_{\mathrm{s}}$ \\
\hline 2011 Jul 9.949 & 0.051 & 317 & 20.76 & 20.53 & 20.32 & 20.49 & 720 & 20.03 & 19.02 & 18.79 \\
\hline 2011 Jul 10.009 & 0.111 & 3871 & 24.26 & 24.57 & 23.83 & 23.61 & 3360 & 20.83 & 20.00 & 19.27 \\
\hline $2011 \mathrm{Jul} 10.045$ & 0.147 & 1133 & 22.61 & 22.80 & 22.39 & 21.12 & 1920 & 19.83 & 18.83 & 18.66 \\
\hline $2011 \mathrm{Jul} 10.003^{\mathrm{a}}$ & 0.105 & 5321 & 23.63 & 23.89 & 23.28 & 23.09 & 6000 & 21.16 & 19.83 & 19.40 \\
\hline
\end{tabular}

Notes. Date specifies the mid-time of the observations. All magnitudes are upper limits in the AB system, uncorrected for Galactic extinction.

${ }^{a}$ Results from stacking all three epochs.

$\beta_{\text {NIRX }} \lesssim 0.15$. In comparison to the X-ray spectral index we find $\beta_{\mathrm{OX}}-\beta_{\mathrm{X}} \lesssim-1.7$ and $\beta_{\mathrm{NIRX}}-\beta_{\mathrm{X}} \lesssim-1.05$. Thus, GRB $111215 \mathrm{~A}$ is a dark burst in the optical and near-IR. As in the case of GRB 110709B, the absence of optical/near-IR emission can be due to dust extinction, or alternatively to a high redshift, $z \gtrsim 18$ based on the $K_{\mathrm{s}}$-band non-detection.

\subsection{JVLA Centimeter Observations}

\subsubsection{GRB $110709 B$}

We observed GRB 110709B with the NRAO Karl G. Jansky Array (JVLA) beginning on 2011 July 11.97 UT $(\delta t \approx 2.1$ days) at a mean frequency of $5.8 \mathrm{GHz}$ and detected a single, unresolved radio source within the XRT error circle. This detection provided the first accurate position for the burst. Follow-up observations demonstrated that the source initially brightened and subsequently faded away, establishing it as the radio afterglow of GRB 110709B.

All observations utilized the WIDAR correlator (Perley et al. 2011 b) with $\sim 2 \mathrm{GHz}$ bandwidth. We calibrated and analyzed the data using standard procedures in the Astronomical Image Processing System (AIPS; Greisen 2003). We excised edge channels and channels affected by radio frequency interference, reducing the effective bandwidth by $\sim 25 \%$ at $5.8 \mathrm{GHz}$. Due to the low declination of the source, we also excised data when $>2 \mathrm{~m}$ of a given antenna was shadowed by another antenna. For the observation at $21.8 \mathrm{GHz}$, we performed reference pointing at $8.4 \mathrm{GHz}$ and applied the pointing solutions, per standard high frequency observing procedures. We observed 3C286 for band-pass and flux calibration and interleaved observations of $\mathrm{J} 1048-1909$ for gain calibration every $3 \mathrm{~m}$ at $21.8 \mathrm{GHz}$ and $\mathrm{J} 1112-2158$ every $4 \mathrm{~m}$ at $5.8 \mathrm{GHz}$. The resulting flux densities at 5.8 and $21.8 \mathrm{GHz}$ are listed in Table 2. The uncertainties are $1 \sigma$ statistical errors, and we note an additional uncertainty in the absolute flux scaling of $\sim 5 \%$. The $5.8 \mathrm{GHz}$ light curve is shown in Figure 1.

Finally, to determine the position of the radio afterglow we fit the source in each image with a Gaussian profile (AIPS task JMFIT), and calculated the mean radio position weighted by the resulting statistical uncertainties. We find a weighted mean position of R.A. $=10^{\mathrm{h}} 58^{\mathrm{m}} 37^{\mathrm{s}} .113( \pm 0.001)$, decl. $=$ $-23^{\circ} 27^{\prime} 16^{\prime \prime} .76( \pm 0.02)$.

\subsubsection{GRB 111215A}

We observed GRB 111215A with the JVLA beginning on 2011 December 17.00 UT $(\delta t \approx 1.4$ days), and subsequently detected the radio afterglow starting at $\delta t \approx 3.35$ days. Observations between 1.4 and 140 days were obtained at mean
Table 2

JVLA Observations of GRB 110709B

\begin{tabular}{lccc}
\hline \hline UT Date & $\begin{array}{c}\delta t \\
(\text { days })\end{array}$ & $\begin{array}{c}v \\
(\mathrm{GHz})\end{array}$ & $\begin{array}{c}F_{v} \\
(\mu \mathrm{Jy})\end{array}$ \\
\hline 2011 Jul 11.97 & 2.07 & 5.8 & $190 \pm 14$ \\
2011 Jul 12.92 & 3.02 & 5.8 & $250 \pm 27$ \\
2011 Jul 16.88 & 6.98 & 5.8 & $310 \pm 18$ \\
2011 Jul 21.97 & 12.07 & 5.8 & $210 \pm 14$ \\
2011 Jul 29.96 & 20.06 & 5.8 & $98 \pm 17$ \\
2011 Aug 19.85 & 40.59 & 5.8 & $98 \pm 6$ \\
2011 Sep 17.70 & 69.72 & 5.8 & $<38^{\mathrm{a}}$ \\
\hline 2011 Jul 22.01 & 12.11 & 21.8 & $<135$ \\
\hline
\end{tabular}

Notes. Dates specify the mid-time of the observations.

${ }^{\text {a }}$ Upper limits are $3 \sigma$.

frequencies of 5.8, 8.4, and $21.8 \mathrm{GHz}$. We used 3C48 for band-pass and flux calibration and interleaved observations of $\mathrm{J} 2311+3425$ every $\sim 4 \mathrm{~m}$ for gain calibration at $21.8 \mathrm{GHz}$ and $\mathrm{J} 2340+2641$ every $\sim 5.5 \mathrm{~m}$ for gain calibration at 5.8 and $8.4 \mathrm{GHz}$. The data were analyzed in the same manner described above, and the resulting flux densities are listed in Table 3. The light curves are shown in Figures 2 and 3.

We obtain a weighted mean position from all $21.8 \mathrm{GHz}$ detections of R.A. $=23^{\mathrm{h}} 18^{\mathrm{m}} 13^{\mathrm{s}} .314( \pm 0.004)$, decl. $=+32^{\circ} 29^{\prime} 39^{\prime \prime} .07$ $( \pm 0.06)$. The JVLA data (along with CARMA observations: Section 2.5) provide the only sub-arcsecond position for GRB 111215A.

\subsection{Millimeter Observations}

\subsubsection{GRB 110709B}

GRB 110709B was observed at a frequency of $345 \mathrm{GHz}$ with the Large APEX Bolometer Camera (LABOCA; Siringo et al. 2009) on the Atacama Pathfinder Experiment (APEX) telescope beginning on 2011 July 11.90 UT ( $\delta t \approx 2.0$ days). No submillimeter counterpart was detected to a $3 \sigma$ upper limit of $\lesssim 6.9$ mJy (de Ugarte Postigo et al. 2011).

\subsubsection{GRB 111215A}

We observed and detected GRB 111215A with the Combined Array for Research in Millimeter Astronomy (CARMA) beginning on 2011 December $16.97 \mathrm{UT}(\delta t \approx 1.38$ days) at $93 \mathrm{GHz}$ and we continued monitoring the afterglow at this frequency for $\approx 16$ days; see Table 3 . We used Neptune as the primary flux calibrator, and 3C454.3 as our band-pass calibrator, and 2236+284 and $2203+317$ as gain calibrators. The overall uncertainty in 
Table 3

Radio Observations of GRB 111215A

\begin{tabular}{|c|c|c|c|c|}
\hline Telescope & UT Date & $\begin{array}{c}\delta t \\
\text { (days) }\end{array}$ & $\begin{array}{c}v \\
(\mathrm{GHz})\end{array}$ & $\begin{array}{c}F_{v} \\
(\mu \mathrm{Jy})\end{array}$ \\
\hline \multirow[t]{30}{*}{ JVLA } & \multirow[t]{2}{*}{2011 Dec 17.00} & \multirow[t]{2}{*}{1.41} & 4.9 & $<115^{\mathrm{a}}$ \\
\hline & & & 6.7 & $<93$ \\
\hline & \multirow[t]{2}{*}{2011 Dec 23.05} & \multirow[t]{2}{*}{7.46} & 4.9 & $470 \pm 28$ \\
\hline & & & 6.7 & $460 \pm 34$ \\
\hline & \multirow{2}{*}{2011 Dec 29.03} & \multirow{2}{*}{13.44} & 4.9 & $300 \pm 21$ \\
\hline & & & 6.7 & $540 \pm 19$ \\
\hline & \multirow[t]{2}{*}{2011 Dec 31.00} & \multirow[t]{2}{*}{15.41} & 4.9 & $820 \pm 30$ \\
\hline & & & 6.7 & $1180 \pm 30$ \\
\hline & \multirow[t]{2}{*}{ 2012 Jan 10.99} & \multirow[t]{2}{*}{26.40} & 4.9 & $590 \pm 33$ \\
\hline & & & 6.7 & $820 \pm 36$ \\
\hline & \multirow[t]{2}{*}{2012 Jan 29.84} & \multirow[t]{2}{*}{45.25} & 4.9 & $420 \pm 52$ \\
\hline & & & 6.7 & $400 \pm 55$ \\
\hline & \multirow[t]{2}{*}{2012 Mar 12.82} & \multirow[t]{2}{*}{88.23} & 4.9 & $330 \pm 39$ \\
\hline & & & 6.7 & $300 \pm 34$ \\
\hline & 2011 Dec 31.00 & 15.41 & 8.4 & $1340 \pm 63$ \\
\hline & 2012 Jan 10.99 & 26.40 & 8.4 & $880 \pm 120$ \\
\hline & 2012 Jan 29.84 & 45.25 & 8.4 & $470 \pm 81$ \\
\hline & 2012 Mar 12.82 & 88.23 & 8.4 & $240 \pm 95$ \\
\hline & \multirow[t]{2}{*}{2011 Dec 18.93} & \multirow[t]{2}{*}{3.35} & 19.1 & $1520 \pm 74$ \\
\hline & & & 24.4 & $1990 \pm 47$ \\
\hline & \multirow[t]{2}{*}{2011 Dec 27.08} & \multirow[t]{2}{*}{11.49} & 19.1 & $1670 \pm 47$ \\
\hline & & & 24.4 & $1650 \pm 74$ \\
\hline & \multirow[t]{2}{*}{2011 Dec 31.00} & \multirow[t]{2}{*}{15.41} & 19.1 & $1530 \pm 49$ \\
\hline & & & 24.4 & $1370 \pm 62$ \\
\hline & \multirow[t]{2}{*}{2012 Jan 10.99} & \multirow[t]{2}{*}{26.40} & 19.1 & $840 \pm 65$ \\
\hline & & & 24.4 & $730 \pm 78$ \\
\hline & \multirow[t]{2}{*}{2012 Jan 29.84} & 45.25 & 19.1 & $500 \pm 66$ \\
\hline & & & 24.4 & $460 \pm 69$ \\
\hline & 2012 Mar 12.82 & 88.23 & 19.1 & $214 \pm 66$ \\
\hline & & & 24.4 & $180 \pm 72$ \\
\hline CARMA & 2011 Dec 17.05 & 1.46 & 93 & $2300 \pm 200$ \\
\hline & 2011 Dec 20.02 & 4.43 & 93 & $1900 \pm 220$ \\
\hline & 2011 Dec 22.04 & 6.45 & 93 & $1900 \pm 220$ \\
\hline & 2011 Dec 24.10 & 8.51 & 93 & $1400 \pm 180$ \\
\hline & 2011 Dec 26.01 & 10.42 & 93 & $1400 \pm 270$ \\
\hline & 2012 Jan 1.14 & 16.55 & 93 & $940 \pm 200$ \\
\hline $\mathrm{PdBI}$ & 2011 Dec 26.67 & 11.08 & 94.5 & $1378 \pm 65$ \\
\hline & 2011 Dec 27.56 & 11.99 & 86.7 & $1356 \pm 69$ \\
\hline & 2012 Jan 1.78 & 17.19 & 104.7 & $799 \pm 70$ \\
\hline & 2012 Jan 9.68 & 24.09 & 86.7 & $644 \pm 94$ \\
\hline & 2012 Jan 12.65 & 27.06 & 86.7 & $478 \pm 58$ \\
\hline & 2012 Jan 26.49 & 40.91 & 86.7 & $<264^{\mathrm{b} 1}$ \\
\hline & 2012 Feb 27.76 & 73.17 & 93.7 & $<294^{\mathrm{b} 2}$ \\
\hline & 2012 Feb 28.53 & 73.94 & 93.7 & $<189^{\mathrm{b} 3}$ \\
\hline SMA & 2011 Dec 18.30 & 2.71 & 230 & $<2600$ \\
\hline
\end{tabular}

Notes. Dates specify the mid-time of the observations. $1 \sigma$ errors are statistical and do not incorporate uncertainty in overall flux scaling.

${ }^{\text {a }}$ Upper limits are $3 \sigma$. Formal measurements are $197 \pm 88 \mu \mathrm{Jy}$ (b1), $9 \pm 98 \mu \mathrm{Jy}$ (b2), and $161 \pm 63 \mu \mathrm{Jy}$ (b3).

the absolute flux calibration is $\sim 20 \%$. We calibrated and visualized the data with the MIRIAD software package (Sault et al. 1995), and measured the flux densities with AIPS for consistency with the JVLA observations (Table 3). We fit the position of the detected $\mathrm{mm}$ afterglow in each epoch with a Gaussian fixed to the beam size, and compute the mean position, weighted by the uncertainty in each epoch, leading to R.A. = $23^{\mathrm{h}} 18^{\mathrm{m}} 13.321( \pm 0.007)$ and decl. $=+32^{\circ} 29^{\prime} 39^{\prime \prime} .04( \pm 0.07)$. This position is consistent with the Swift/X-ray position.

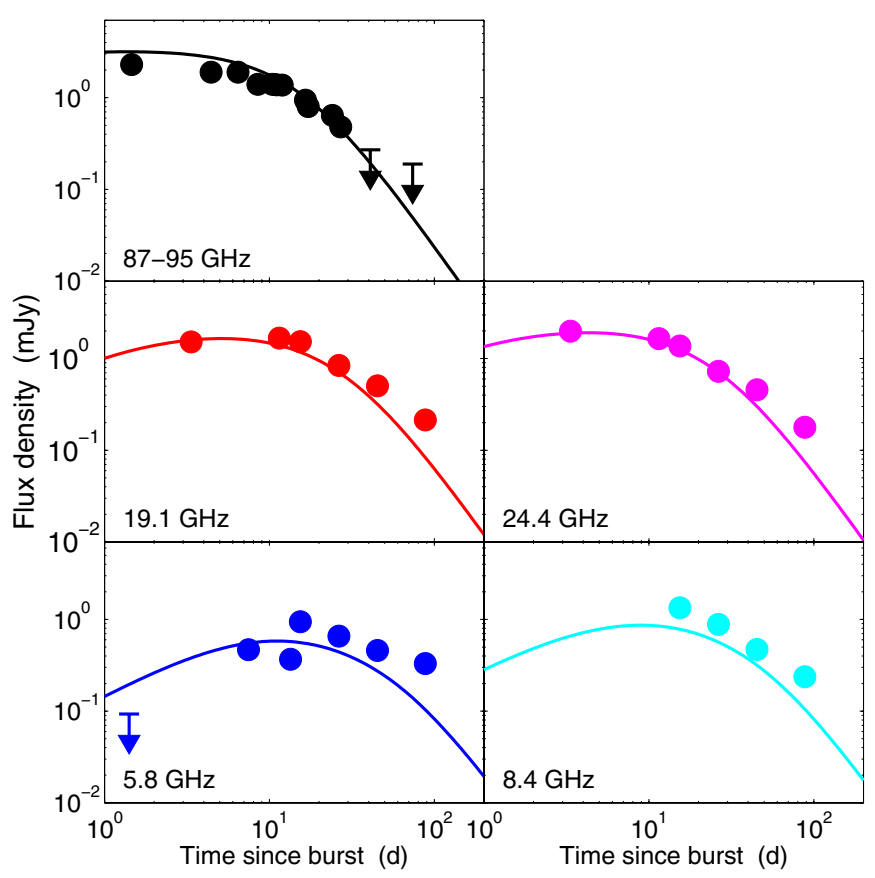

Figure 3. Radio light curves of GRB 111215A at 5.8, 8.4, 19.1, 24.4, and $87-95 \mathrm{GHz}$. Afterglow model fits are shown with the solid lines. The derived afterglow parameters are listed in Table 5.

(A color version of this figure is available in the online journal.)

We also observed GRB 111215A with the IRAM Plateau de Bure interferometer (PdBI) on 2011 December 26.67 ( $\delta t \approx$ 11.1 days) at $94.5 \mathrm{GHz}$ under excellent weather conditions in dual polarization with the $3.6 \mathrm{GHz}$ bandwidth of the WIDEX correlator, detecting the afterglow at a flux level (1380 \pm $60 \mu \mathrm{Jy}$ ) consistent with our CARMA observations. We utilized MWC349, 3C454.3, and J2253+326 for flux, band-pass and gain calibration, respectively. We continued observations with PdBI over the course of two months until the source faded below the detection threshold. In the first two epochs of observations when the source was very bright $(\mathrm{S} / \mathrm{N} \sim 20)$, we checked for flux variability. We report no evidence for significant flaring (as found in another dark burst, GRB 051022; Castro-Tirado et al. 2007): the flux was constant within $1.5 \sigma$ and $1.0 \sigma$ error limits on 2011 December 26.67 and 27.56, respectively. All calibration and image processing were performed with standard procedures in the Grenoble Image and Line Data Analysis Software (GILDAS) package. ${ }^{19}$ The overall uncertainty in flux calibration for PdBI is $\sim 10 \%-20 \%$. The CARMA and PdBI observations utilized different gain calibrators, but both had observations of 3C454.3 for band-pass calibration, giving us confidence in the relative flux scaling (4.3 Jy at CARMA on December 26.01 at $93 \mathrm{GHz}$ and $4.39 \mathrm{Jy}$ at PdBI on December 26.67 at $94.5 \mathrm{GHz}$ ). The millimeter data are summarized in Table 3 and the first panel of Figure 3.

We observed GRB 111215A with the Submillimeter Array (SMA; Ho et al. 2004) beginning on 2011 December 18.15 UT $(\delta t \approx 2.7$ days) at $230 \mathrm{GHz}$ with $8 \mathrm{GHz}$ bandwidth. We performed gain calibration using $\mathrm{J} 2311+344$ and $\mathrm{J} 2236+284$, band-pass calibration using $3 \mathrm{C} 84$, and flux calibration using real-time measurements of the system temperatures, with observations of Uranus and Callisto to set the overall flux scale (accurate to about 10\%). We calibrated the data using the MIR

\footnotetext{
19 http://www.iram.fr/IRAMFR/GILDAS
} 

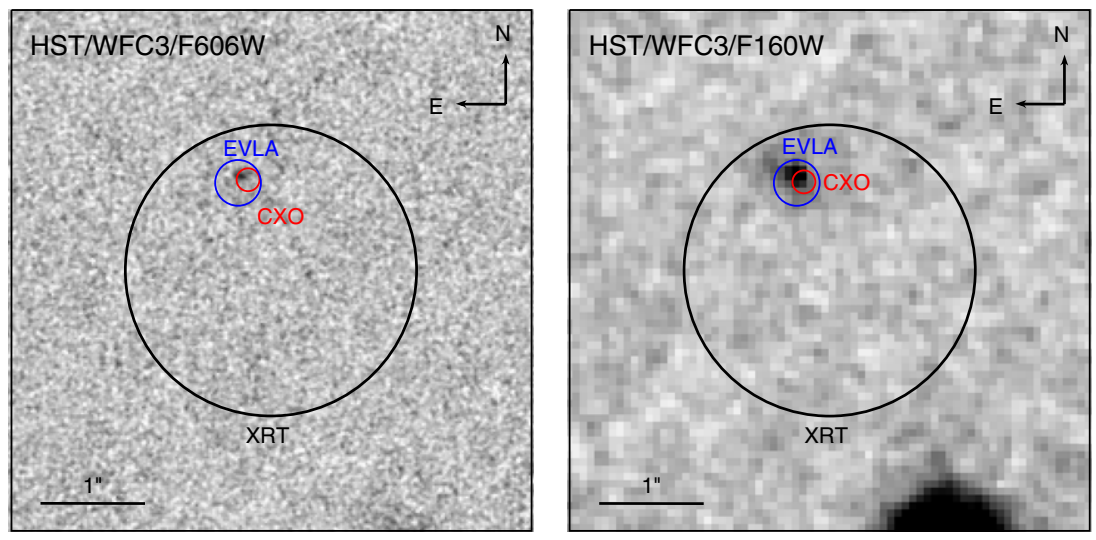

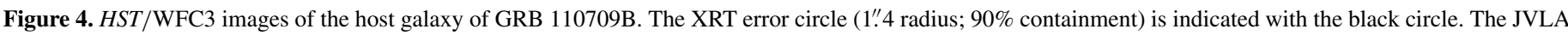

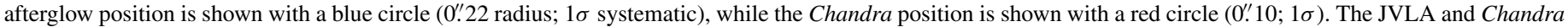
positions coincide with a galaxy that we consider to be the host of GRB 110709B.

(A color version of this figure is available in the online journal.)

software package, and analyzed and imaged the observation with MIRIAD. We do not detect the GRB afterglow to a $3 \sigma$ limit of $\lesssim 2.6 \mathrm{mJy}$.

\subsection{Host Galaxy Observations and Redshift Constraints}

\subsubsection{GRB 110709B}

We obtained Hubble Space Telescope (HST) observations of GRB 110709B with the Wide Field Camera 3 (WFC3; Kimble et al. 2008) on 2011 November 8.94 UT ( $\delta t \approx 122$ days) using the ultraviolet imaging spectrograph (UVIS) with the F606W filter, and on 2011 November 12.94 UT ( $\delta t \approx 126$ days) using the infrared (IR) channel with the F160W filter (PI: Levan). One orbit of observations was obtained in each filter, with exposure times of $2610 \mathrm{~s}$ (F606W) and $2480 \mathrm{~s}$ (F160W). The data were processed using multidrizzle (Fruchter \& Hook 2002; Koekemoer et al. 2002) with output pixel scales set at $0 ! 02$ and 0.07 , respectively.

To locate the absolute radio and X-ray afterglow positions on the HST images we tie the F606W and F160W images to the 2MASS reference frame using a wider field Gemini-South GMOS $r$-band image as an intermediary. Using 20 common sources with 2MASS, the absolute astrometry of the Gemini image has an rms scatter of $\sigma_{\mathrm{GMOS}-2 \mathrm{MASS}}=0^{\prime} .15$ in each coordinate. The HST images are tied to the GMOS image using 30 common sources with a resulting rms scatter of $\sigma_{H S T \text {-GMOS }}=00^{\prime} 03$ in each coordinate. We further refine the Chandra afterglow position relative to the $H S T$ astrometric frame using a single common source. This leads to a shift in the $\mathrm{X}$-ray position of $\delta$ R.A. $=-0^{\prime} \cdot 10\left(-0^{\prime \prime} \cdot 07\right)$ and $\delta$ decl. $=+0^{\prime \prime} \cdot 20$ $\left(+0{ }^{\prime} 18\right)$ relative to the F606W (F160W) reference frame. There are no common sources between the HST and JVLA images.

The resulting uncertainty in the radio afterglow position in the HST reference frame is dominated by the absolute astrometry, and corresponds to a radius of $0^{\prime} .22(1 \sigma$; the centroid uncertainty of the radio position is only $0{ }^{\prime} 02$ in each coordinate). The uncertainty in the X-ray afterglow position is reduced by the relative tie of the Chandra and HST reference frames, leading to a radius of 0 ' $10(1 \sigma$; the centroid uncertainty of the Chandra position is about $0{ }^{\prime} 08$ in each coordinate). Within the uncertainty regions we find a single galaxy in both HST images (Figure 4), which we identify as the host of GRB 110709B. Photometry of this source using the tabulated
WFC3 zero points gives $m_{\mathrm{F} 606 \mathrm{~W}}=26.78 \pm 0.17 \mathrm{AB}$ mag and $m_{\mathrm{F} 160 \mathrm{~W}}=25.13 \pm 0.10 \mathrm{mag}$, corrected for Galactic extinction.

To determine the probability of chance coincidence, $P_{\mathrm{cc}}(<\delta R)=1-\exp \left[-\pi(\delta R)^{2} \sigma(\leqslant m)\right]$, we use the galaxy number counts, $\sigma(\leqslant m)=10^{0.33(m-24)-2.32} \operatorname{arcsec}^{-2}$ (Hogg et al. 1997; Beckwith et al. 2006). For the XRT error circle we infer $P_{c c} \approx 0.1$, while for the more precise JVLA and Chandra positions we find $P_{c c} \approx 0.006$ and $\approx 0.001$, respectively. Thus, the sub-arcsecond radio and $\mathrm{X}$-ray positions enable a robust association with the galaxy, while the XRT position alone would have led to marginal confidence. This is essential since the detection of the host galaxy in the F606W filter limits the redshift to $z \lesssim 4$, indicating that GRB $110709 \mathrm{~B}$ is dark due to extinction and not a $z \gtrsim 18$ origin.

For a redshift from $z \sim 1$, the F606W band samples the rest-frame UV emission from the host galaxy. Based on the observed flux density calculated from the apparent magnitude $\left(m_{\mathrm{F} 606 \mathrm{~W}}=26.78 \pm 0.17 \mathrm{AB} \mathrm{mag}\right)$ assuming the standard cosmological parameters noted in Section 1 to calculate the luminosity distance (Wright 2006), we utilize Equation (1) in Kennicutt (1998)

$$
\operatorname{SFR}\left(M_{\odot} \mathrm{yr}^{-1}\right)=1.4 \times 10^{-28} L_{v}\left(\mathrm{erg} \mathrm{s}^{-1} \mathrm{~Hz}^{-1}\right)
$$

to infer SFR $\approx 0.3-3.2 M_{\odot} \mathrm{yr}^{-1}$ for $z=1-4$, not corrected for possible galactic-scale extinction.

\subsubsection{GRB 111215A}

We obtained $i$-band observations of GRB 111215A with GMOS on the Gemini-North $8 \mathrm{~m}$ telescope on 2011 December $23.21 \mathrm{UT}(\delta t \approx 7.6$ days), with a total exposure time of $2160 \mathrm{~s} \mathrm{in}$ 0 '.9 seeing. Astrometric matching to the 2MASS catalog using 30 common sources reveals a galaxy within the enhanced XRT error circle at R.A. $=23^{\mathrm{h}} 18^{\mathrm{m}} 13^{\mathrm{s}} \cdot 317$, decl. $=+32^{\circ} 29^{\prime} 38^{\prime \prime} .77$, with an uncertainty of about $00^{\prime} 16$ in each coordinate (absolute) and an uncertainty of about $0^{\prime \prime} .06$ in the galaxy centroid; see Figure 5. We obtained imaging observations with the LowResolution Imaging Spectrometer (LRIS; Oke et al. 1995) on the Keck I $10 \mathrm{~m}$ telescope on 2012 July $15.20 \mathrm{UT}$ ( $\delta t=212.6$ days) in the $g$ - and $I$-band filters. Finally, we also obtained observations of the GRB 111215A host galaxy with the OSIRIS instrument (Sánchez et al. 2012) on the $10.4 \mathrm{~m}$ Gran Telescopio Canarias (GTC) on 2012 August 23.02 UT in the griz bands with an average seeing of 0.9 . 


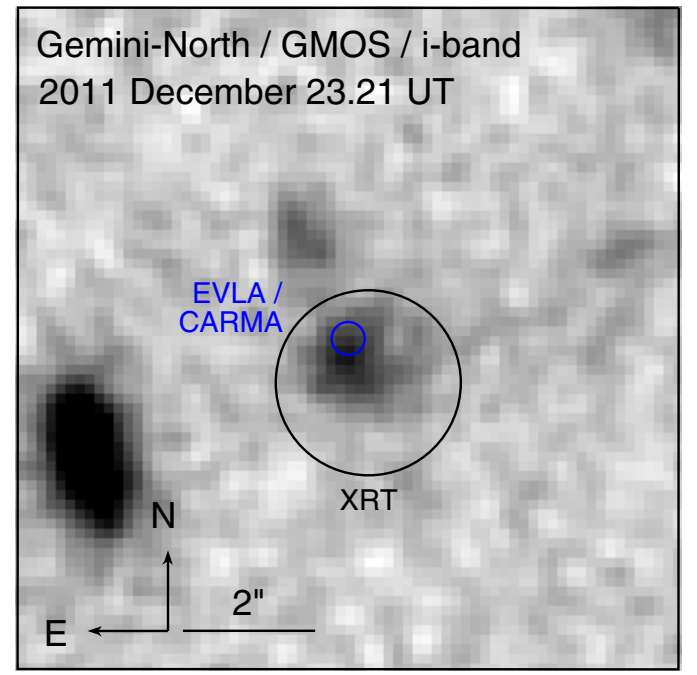

Figure 5. Gemini/GMOS $i$-band image of the host galaxy of GRB 111215A. The XRT error circle (1". 4 radius; $90 \%$ containment) is indicated with the black circle. The JVLA and CARMA afterglow positions are shown with a blue circle $(0.13 ; 1 \sigma$ systematic) coinciding with a galaxy that we consider to be the host of GRB 111215A

(A color version of this figure is available in the online journal.)

Taking into account the uncertainty in the JVLA radio afterglow position ( $0^{\prime}$.06 in each coordinate), the resulting overall uncertainty in the location of the afterglow on the Gemini image is 0 '.26 radius $(1 \sigma)$. Similarly, the uncertainty in the CARMA mm afterglow position ( 0 '.08 in each coordinate) leads to an overall uncertainty of 0.27 radius $(1 \sigma)$. The resulting offset between the host centroid and radio positions is 0 "' $30 \pm 0.26$.

Photometry of the galaxy relative to the Sloan Digital Sky Survey catalog results in a brightness of $m_{g}=24.40 \pm 0.18$, $m_{r}=24.24 \pm 0.15, m_{i}=23.87 \pm 0.18$, and $m_{z}=23.90 \pm 0.36$ (GTC), and $m_{g}=24.50 \pm 0.15 \mathrm{mag}$ and $m_{i}=23.64 \pm 0.05 \mathrm{mag}$ corrected for Galactic extinction (Gemini). The probability of chance coincidence for this galaxy within the XRT error circle is $P_{\mathrm{cc}} \approx 0.01$, with a slightly lower probability of $\approx 7 \times 10^{-3}$ using the radio position, dominated by the physical extent of the galaxy of about $1^{\prime \prime}$. Given the low chance coincidence probability we consider this galaxy to be the host of GRB 111215A.

To determine the redshift of the host we obtained an $1800 \mathrm{~s}$ spectrum with the Low Resolution Imaging Spectrometer (LRIS; Oke et al. 1995; Rockosi et al. 2010) on Keck I using a 0.7 slit with the 400/3400 grism in the blue arm and the $400 / 8500$ grating in the red arm, providing effectively continuous wavelength coverage from the atmospheric cutoff to $10280 \AA$ (with a total throughput within $50 \%$ of peak to about $10030 \AA$ ). The slit was oriented to cover the host galaxy, as well as the fainter extended source to the northeast. The spectrum was reduced using standard techniques implemented in a custom pipeline.

We detect continuum emission from both galaxies on the blue side, and from the host galaxy on the red side. We identify only one emission feature from the nearby galaxy, a marginally resolved line centered at $8019 \AA$. Interpreted as the [O II] $\lambda 3727$ doublet, this indicates a redshift ${ }^{20}$ of $z=1.152$. We do not identify any emission lines from the host galaxy. Given that the galaxy is reasonably bright in $g$ - and $i$-band and is expected to

\footnotetext{
20 We also examined the possibility of alternative line identifications and redshifts, but these would imply the detection of other spectral lines elsewhere that are not observed.
}

be actively star-forming, the lack of emission lines indicates that $z \gtrsim 1.8$ (from the non-detection of [O II] 33727 to $10030 \AA$ ) and $z \lesssim 2.9$ (from the absence of a break due to the Lyman limit to $3860 \AA){ }^{21}$

At $z \sim 1.8-2.9$, the observed $i$ - and $g$-band fluxes trace the host galaxy rest-frame UV emission. The observed color of $g-i \approx 0.85 \pm 0.20 \mathrm{mag}$ is indicative of extinction; for example, it is well-matched to the observed color of Arp 220, a local ULIRG. Using the observed $i$-band flux density, which is less susceptible to extinction corrections, we infer SFR $\approx$ 15-30 $M_{\odot} \mathrm{yr}^{-1}(z=1.8-2.9)$, which should be considered as a minimum value due to the uncertain extinction.

\section{AFTERGLOW MODELING AND REST-FRAME EXTINCTION}

GRBs 110709B and 111215A are dark in both the optical and near-IR bands due to rest-frame dust extinction; a high-redshift origin is ruled out by the association with optically-detected host galaxies. It is also of note that for extreme redshifts $(z>18)$, the $N_{\mathrm{H} \text {,int }}$ implied by the observed excess in the X-ray spectra would be very high (e.g., $>10^{24} \mathrm{~cm}^{-2}$ ), further supporting the rejection of an extreme redshift for either event. In addition to providing accurate localizations and hence secure host associations, the radio data also allow us to determine the properties of the bursts and their local environments. This not only provides a more robust measure of the required rest-frame extinction than using $\beta_{\mathrm{OX}}$ alone (which requires an assumption about the location of the synchrotron cooling frequency), but it also allows us to compare the explosion properties of dark and optically-bright bursts.

We model the radio and X-ray data for GRBs 110709B and $111215 \mathrm{~A}$ using the standard afterglow synchrotron model (Granot \& Sari 2002; Sari et al. 1999) to (1) determine the expected optical and near-IR brightness, and hence the required level of rest-frame extinction given the observed limits; and (2) determine the properties of the bursts and their circumburst environments. We follow the standard assumptions of synchrotron emission from a power-law distribution of electrons $\left(N(\gamma) \propto \gamma^{-p}\right.$ for $\left.\gamma \geqslant \gamma_{m}\right)$ with constant fractions of the post-shock energy density imparted to the electrons $\left(\epsilon_{e}\right)$ and magnetic fields $\left(\epsilon_{B}\right)$. The additional free parameters of the model are the isotropic-equivalent blast-wave kinetic energy $\left(E_{\mathrm{K} \text {,iso }}\right)$, the circumburst density (parameterized as $n$ for a constant density medium: ISM; or as $A$ for a wind medium with $\left.\rho(r)=A r^{-2}\right)$, and a jet break time $\left(t_{j}\right)$. To determine the opening angle $\left(\theta_{j}\right)$, we use the conversions from $t_{j}$ given by Sari et al. (1999) and Chevalier \& Li (2000), with the appropriate dependence on $E_{\mathrm{K} \text {,iso }}$ and the circumburst density ( $n$ or $A$, respectively).

Using the time evolution of the synchrotron spectrum for both the interstellar medium (ISM) and wind density profiles in the pre- and post-jet break phases (Chevalier \& Li 2000; Granot \& Sari 2002) we simultaneously fit all X-ray and radio observations for each burst. The resulting best-fit parameters are summarized in Tables 4 and 5 using redshifts of $z=1,2,3,4$ for GRB 110709B and $z=2$ for GRB 111215A. In both cases we find that the most stringent constraint on the restframe extinction are provided by the observed $K$-band limits. We use the Small Magellanic Cloud (SMC) extinction curve to

\footnotetext{
21 The trace is detected to $3400 \AA$, corresponding to $z \lesssim 2.7$, but only at $\sim 1 \sigma$
} Hence, we utilize the more conservative redshift constraint of 2.9 . 
Table 4

Results of Broadband Afterglow Modeling for GRB 110709B

\begin{tabular}{lllll}
\hline \hline Parameter & $z=1$ & $z=2$ & $z=3$ & $z=4$ \\
\hline$E_{\mathrm{K}, \text { iso,52 }(\mathrm{erg})}$ & 0.5 & 2.8 & 7.1 & 13.2 \\
$A^{*}\left(5 \times 10^{11} \mathrm{~g} \mathrm{~cm}^{-1}\right)$ & 4.0 & 5.7 & 7.2 & 8.5 \\
$\bar{\epsilon}_{e}$ & 0.18 & 0.11 & 0.08 & 0.07 \\
$\epsilon_{B}$ & 0.002 & 0.002 & 0.002 & 0.002 \\
$p$ & 2.07 & 2.06 & 2.05 & 2.05 \\
$t_{j}$ (days) & 3.65 & 3.45 & 3.27 & 3.11 \\
$\theta_{j}(\mathrm{rad})$ & 0.40 & 0.25 & 0.19 & 0.16 \\
$E_{\mathrm{K}, 51}(\mathrm{erg})$ & 0.4 & 0.9 & 1.3 & 1.7 \\
$E_{\gamma, \text { iso,52 }}{ }^{\mathrm{a}}(\mathrm{erg})$ & 7.0 & 26 & 52 & 83 \\
$E_{\gamma, 51}(\mathrm{erg})$ & 5.4 & 8.0 & 9.4 & 10.6 \\
$A_{V}{ }^{\mathrm{b}}(\mathrm{mag})$ & $\gtrsim 10.5$ & $\gtrsim 5.3$ & $\gtrsim 4.4$ & $\gtrsim 3.4$ \\
\hline
\end{tabular}

Notes. See Section 3 for description of model parameters.

a Using the Konus-WIND 20-5000 keV fluence.

${ }^{\mathrm{b}}$ Using the SMC extinction curve.

Table 5

Results of Broadband Afterglow Modeling for GRB 111215A

\begin{tabular}{lcc}
\hline \hline Parameter & $z=2$ & $z=3$ \\
\hline$E_{\mathrm{K}, \text { iso,52 }(\mathrm{erg})}$ & 7.7 & 13.3 \\
$A^{*}\left(5 \times 10^{11} \mathrm{~g} \mathrm{~cm}^{-1}\right)$ & 17 & 19 \\
$\bar{\epsilon}_{e}$ & 0.16 & 0.14 \\
$\epsilon_{B}$ & $9.0 \times 10^{-5}$ & $1.0 \times 10^{-4}$ \\
$p$ & 2.30 & 2.30 \\
$t_{j}($ days $)$ & 12 & 13 \\
$\theta_{j}(\mathrm{rad})$ & 0.35 & 0.30 \\
$E_{\mathrm{K}, 51}(\mathrm{erg})$ & 4.6 & 5.8 \\
$E_{\gamma, \text { iso,52 }}{ }^{\mathrm{a}}(\mathrm{erg})$ & 4.5 & 9.0 \\
$E_{\gamma, 51}(\mathrm{erg})$ & 2.7 & 3.9 \\
$A_{V}{ }^{\mathrm{b}}(\mathrm{mag})$ & $\gtrsim 8.5$ & $\gtrsim 6.8$ \\
\hline
\end{tabular}

Notes. See Section 3 for description of model parameters.

${ }^{a}$ Using the fluence in the Swift/BAT 15-150 keV band.

${ }^{\mathrm{b}}$ Using the SMC extinction curve.

determine the rest-frame extinction, although the Milky Way extinction curve gives similar results.

For GRB 110709B we find that the data favor a wind environment, with a jet break at $t_{j} \approx 3.1-3.6$ days, corresponding to an opening angle, $\theta_{j}, \approx 9^{\circ}-23^{\circ}$; the jet break occurs earlier and the resulting opening angle is narrower as the redshift increases from $z=1$ to $z=4$. The resulting beaming-corrected energies are $E_{K} \approx(0.4-1.7) \times 10^{51} \mathrm{erg}$ and $E_{\gamma} \approx(5.4-10.6) \times 10^{51} \mathrm{erg}$ (in the observed $20-5000 \mathrm{keV}$ fluence of the initial event). The circumburst density is characterized by a mass-loss rate of $\dot{M} \approx(4-8) \times 10^{-5} M_{\odot} \mathrm{yr}^{-1}$ for a wind velocity of $v_{w}=10^{3} \mathrm{~km} \mathrm{~s}^{-1}$ (i.e., $A^{*} \approx 4-8$ ). Finally, using the near-IR $K_{\mathrm{s}}$-band limit we find that the required extinction ranges from $A_{V}^{\text {host }} \gtrsim 10.5 \mathrm{mag}$ at $z=1$ to $\gtrsim 3.5 \mathrm{mag}$ at $z=4$. We note that the best-fit ISM model also requires a high density of $\sim 10-100 \mathrm{~cm}^{-3}$ at $z \sim 1-4$.

For GRB 111215A we again find that a wind environment provides a better fit, with a jet break at $t_{j} \approx 12$ days, corresponding to an opening angle of $\theta_{j} \approx 24^{\circ}$. The resulting beaming-corrected energies are $E_{K} \approx 3.7 \times 10^{51} \mathrm{erg}$ and $E_{\gamma} \approx 3.9 \times 10^{51} \mathrm{erg}$ (in the observed $15-150 \mathrm{keV}$ band). The circumburst density is characterized by a substantial massloss rate of $\dot{M} \approx 2 \times 10^{-4} M_{\odot} \mathrm{yr}^{-1}$ (for a wind velocity of $\left.v_{w}=10^{3} \mathrm{~km} \mathrm{~s}^{-1}\right)$. Finally, using the near-IR $K_{\mathrm{s}}$-band limit we find a required extinction of $A_{V}^{\text {host }} \gtrsim 8.5 \mathrm{mag}$ (for $z=2$ ). We note that the best-fit ISM model also requires a density of

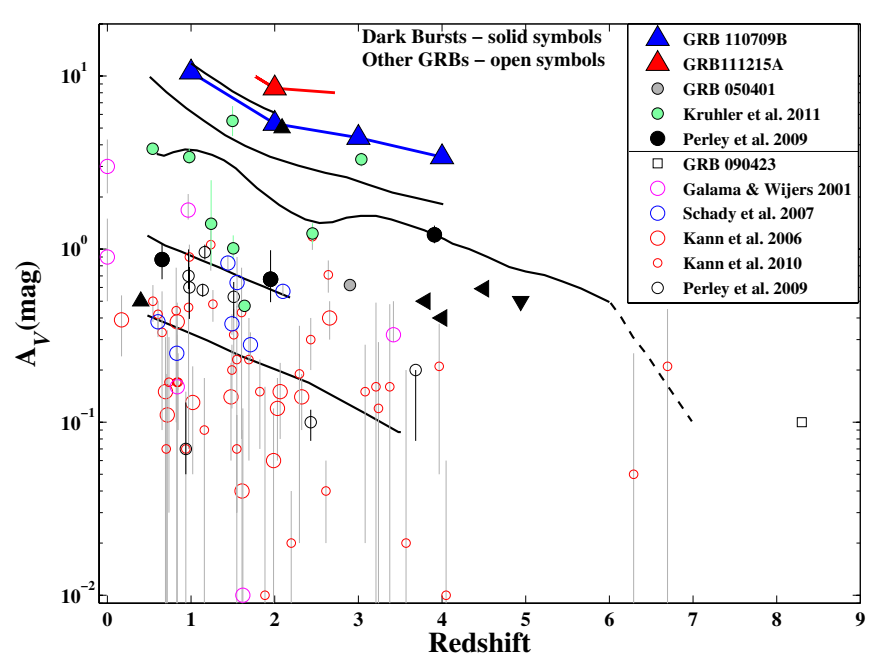

Figure 6. Rest-frame extinction ( $A_{V}^{\text {host }}$ ) vs. redshift for GRBs 110709B (blue triangles) and 111215A (red triangle); for both bursts these are lower limits. Previous dark bursts (see Perley et al. 2009; Krühler et al. 2011; Watson et al. 2006, and references therein) are marked with solid symbols; GRBs 061222, 070306, and 070521 are the three bursts with extinction of at least $\sim 5$ mag (Jaunsen et al. 2008/Krühler et al. 2011; Perley et al. 2009). Triangles indicate limits on $A_{V}^{\text {host }}$ and/or redshift. Allowed values for GRBs without firm redshifts are indicated by black lines (Perley et al. 2009). Optically-bright GRBs are marked with open symbols; these bursts generally have $A_{V}^{\text {host }} \lesssim 1$ mag. GRBs $110709 \mathrm{~B}$ and $111215 \mathrm{~A}$ exhibit some of the highest extinction values to date.

(A color version of this figure is available in the online journal.)

$\sim 100-300 \mathrm{~cm}^{-3}$. Dust extinction this large is indicative of dust associated with the local environment of the GRB (e.g., Reichart \& Price 2002; Campana et al. 2006).

\section{DISCUSSION}

\subsection{Extinction and Neutral Hydrogen Column Density}

The large rest-frame extinction that is required to explain the lack of optical and near-IR emission from GRBs 110709B and $111215 \mathrm{~A}$ is uncommon amongst known GRBs. In Figure 6 we plot the minimum values of $A_{V}^{\text {host }}$ for the two bursts as a function of redshift, along with several comparison samples from the literature of optically-bright bursts and previous dark bursts. In general, optically-bright GRBs have inferred extinction values of $\lesssim 1 \mathrm{mag}$, with a mean of $\sim 0.2-0.3 \mathrm{mag}$ (Kann et al. 2006, 2010; Schady et al. 2007; Perley et al. 2009; Krühler et al. 2011). Dark GRBs span $A_{V}^{\text {host }} \approx 1-6$ mag (Perley et al. 2009; Krühler et al. 2011; Prochaska et al. 2009; Castro-Tirado et al. 2007; Rol et al. 2007), with the largest extinction values of 5-6 mag inferred for GRBs 061222, 070306, and 070521 (Perley et al. 2009; Krühler et al. 2011; Jaunsen et al. 2008). In this context, the extinction values measured here are at the top of the distribution, $A_{V}^{\text {host }} \gtrsim 5.3 \mathrm{mag}(110709 \mathrm{~B})$ and $\gtrsim 8.5 \mathrm{mag}$ (111215A) at $z=2$.

In Figure 7 we plot the intrinsic neutral hydrogen column densities, $N_{\mathrm{H} \text {,int }}$, from our X-ray analysis as a function of redshift, in comparison with the population of all Swift long GRBs with known redshifts (up to 2010 December; Margutti et al. 2013; see also Jakobsson et al. 2006 and Fynbo et al. 2009). We find that GRBs 110709B and 111215A lie at the upper end of the distribution for long GRBs. In particular, at a fiducial redshift of $z=2$ they have $\log \left(N_{\mathrm{H} \text {,int }}\right) \approx 22.1$ and $\approx 22.5$, respectively, in comparison with the median value of all detections and upper limits of $\left\langle\log \left(N_{\mathrm{H}, \text { int }}\right)\right\rangle \approx 21.7$. This result confirms recent suggestions that dark GRBs have systematically larger neutral 


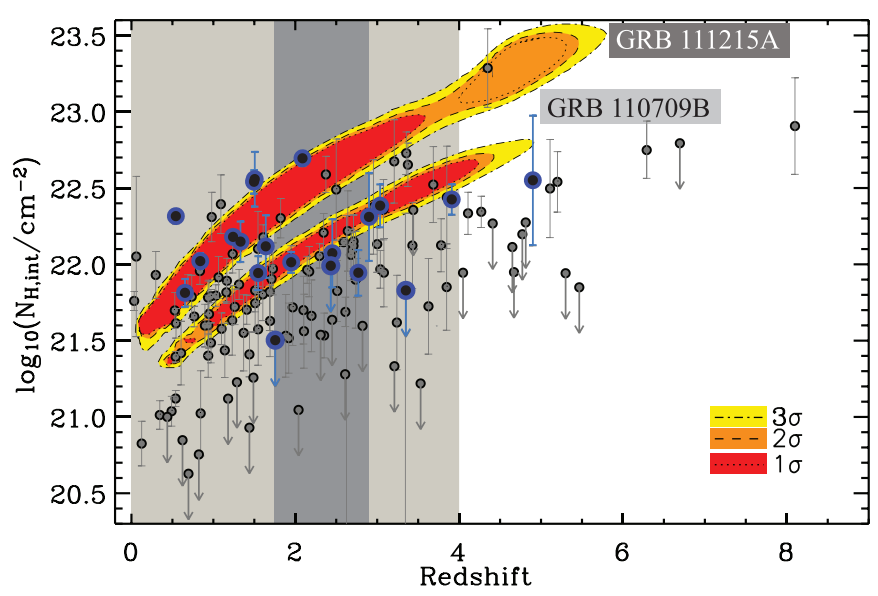

Figure 7. Intrinsic neutral hydrogen column density $\left(N_{\mathrm{H}, \text { int }}\right)$ inferred from X-ray observations vs. redshift for GRBs $110709 \mathrm{~B}$ and $111215 \mathrm{~A}$ (contours). The allowed redshift ranges for each burst are marked by the gray shaded regions. For comparison, we overlay data for long GRBs in the Swift sample up to 2010 December (Margutti et al. 2013); dark bursts are marked by blue/black circles, while optically-bright events are marked by gray circles. GRBs $110709 \mathrm{~B}$ and $111215 \mathrm{~A}$ clearly lie in the upper portion of the $N_{\mathrm{H} \text {,int }}$ distribution of long GRBs, as expected for dark bursts.

(A color version of this figure is available in the online journal.)

hydrogen columns, generally with $\log \left(N_{\mathrm{H}, \text { int }}\right) \gtrsim 22$ (Perley et al. 2009; Campana et al. 2012); see Figure 7. In their spectroscopic sample of GRBs, Fynbo et al. (2009) also find dark bursts to have higher excess absorption in general, and more specifically, the three bursts with highest column densities are dark. Indeed, only a handful of events in the Swift sample have values of $N_{\mathrm{H} \text {,int }}$ comparable to that of GRB 111215A (if $z \gtrsim 2$ ). The same conclusion is true for GRB 110709B if it resides at the upper end of the allowed redshift distribution $(z \sim 3-4)$, although at $z \sim 1$ its neutral hydrogen column is typical of the overall long GRB population.

A correlation between extinction and $N_{\mathrm{H}}$ is known to exist in the Milky Way and the Magellanic Clouds, with $N_{\mathrm{H}} \approx$ $2 \times 10^{21} A_{V} \mathrm{~cm}^{-2}$ (Predehl \& Schmitt 1995; Güver \& Özel 2009; Watson 2011). As shown in Figure 8, optically-bright GRBs generally have lower values of $A_{V}^{\text {host }}$ than would be inferred from this relation (e.g., Galama \& Wijers 2001; Stratta et al. 2004; Zafar et al. 2011), with mean values of $N_{\mathrm{H} \text {,int }} \approx 5 \times 10^{21} \mathrm{~cm}^{-2}$ and $A_{V}^{\text {host }} \approx 0.2-0.3 \mathrm{mag}$; the expected extinction based on the Galactic relation is $A_{V}^{\text {host }} \approx 2.5 \mathrm{mag}$. Dark GRBs also generally have lower extinction than expected, with $N_{\mathrm{H} \text {,int }} \approx$ $(5-50) \times 10^{21} \mathrm{~cm}^{-2}$ and $A_{V}^{\text {host }} \approx 1-5 \mathrm{mag}$, whereas extinctions of $A_{V}^{\text {host }} \approx 5-25$ mag would be expected.

For the bursts presented here we find potentially different results. GRB 111215A agrees with the Galactic relation if it resides at the lower redshift bound $(z \approx 1.8)$, in which case $N_{\mathrm{H} \text {,int }} \approx 2.5 \times 10^{22} \mathrm{~cm}^{-2}$ and $A_{V}^{\text {host }} \gtrsim 11 \mathrm{mag}$. At the higher redshift bound $(z \approx 2.7)$ we find $N_{\mathrm{H}, \text { int }} \approx 5 \times 10^{22} \mathrm{~cm}^{-2}$ and $A_{V}^{\text {host }} \gtrsim 6 \mathrm{mag}$ (with an expected $A_{V}^{\text {host }} \approx 23 \mathrm{mag}$ ). This is still consistent with the Galactic relation since we only place a lower bound on $A_{V}^{\text {host }}$. Similarly, GRB $110709 \mathrm{~B}$ crosses the Galactic relation at $z \approx 2$, with $N_{\mathrm{H} \text {,int }} \approx 1.2 \times 10^{22} \mathrm{~cm}^{-2}$ and $A_{V}^{\text {host }} \gtrsim 5.3$ mag. However, at $z \lesssim 2$ it has larger extinction than expected; for example, at $z=1$ we measure $A_{V}^{\text {host }} \gtrsim 10.5$ mag compared with the expected value of $\approx 2.5 \mathrm{mag}$. At $z \gtrsim 2$ the situation is similar to GRB $111215 \mathrm{~A}$, namely the minimum value of $A_{V}^{\text {host }}$ is below the expected Galactic relation, but this is only a lower limit. We therefore conclude that the dark bursts

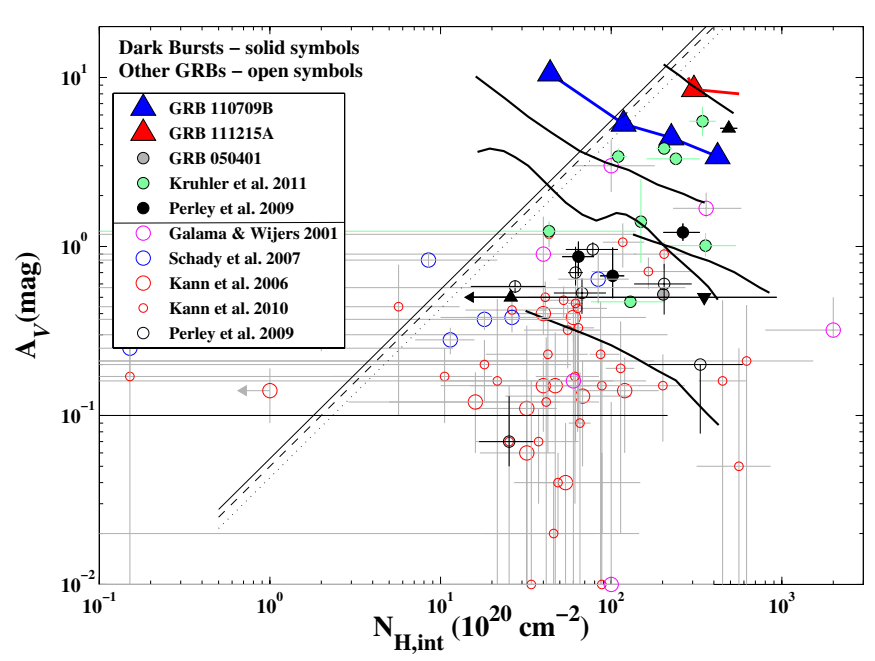

Figure 8. Rest-frame extinction ( $A_{V}^{\text {host }}$ ) vs. intrinsic neutral hydrogen column density $\left(N_{\mathrm{H}, \text { int }}\right)$ inferred from X-ray observations for GRBs $110709 \mathrm{~B}$ (blue triangles) and 111215A (red triangle). For comparison, we plot the same sample of bursts shown in Figure 6. The diagonal lines indicate the empirical $A_{V}-N_{\mathrm{H}}$ relations for the Milky Way and Magellanic Clouds. Most GRBs exhibit lower values of $A_{V}^{\text {host }}$ than expected from the local relations, but GRBs 110709B and $111215 \mathrm{~A}$ may be consistent with these relations.

(A color version of this figure is available in the online journal.)

with the largest known extinction values are potentially in line with the Galactic $N_{\mathrm{H}}-A_{V}$ relation, although they may also reside below the expected correlation in line with the bulk of the GRB population.

\subsection{Explosion and Circumburst Properties of Dark Bursts}

A few previous dark GRBs have been localized to subarcsecond precision in the radio. GRB 970828 was detected in a single radio observation with $F_{v}(8.46 \mathrm{GHz})=147 \pm 33 \mu \mathrm{Jy}$ $(4.5 \sigma)$, but was not detected in observations only 1 day earlier and 2 days later (Djorgovski et al. 2001). This single marginal detection was used to refine the position from the X-ray region of $10^{\prime \prime}$ radius, and to claim an association with a galaxy at $z=0.958$. The inferred extinction at this redshift is $A_{V}^{\text {host }} \gtrsim 3.8$ mag. GRB 000210 was localized to sub-arcsecond precision with Chandra, and was also marginally detected in the single epoch in the radio with $F_{\nu}(8.46 \mathrm{GHz})=93 \pm 21 \mu \mathrm{Jy}$

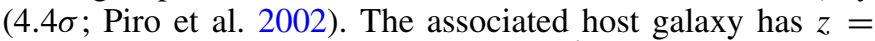
0.846 , and the inferred extinction is $A_{V}^{\text {host }} \approx 0.9-3.2 \mathrm{mag}$. GRB 020819 was detected at high significance in several epochs of radio observations, leading to an association with a face-on spiral galaxy at $z=0.410$ (Jakobsson et al. 2005). The required extinction was a mild $A_{V}^{\text {host }} \approx 0.6-1.5 \mathrm{mag}$. Finally, GRB 051022 was localized using radio and millimeter observations to a host galaxy at $z=0.809$, requiring an extinction of $A_{V}^{\text {host }} \gtrsim 3.5 \mathrm{mag}$ (Castro-Tirado et al. 2007; Rol et al. 2007). Broadband modeling indicates a beaming-corrected energy of $\approx 10^{51} \mathrm{erg}$, and a circumburst density characterized by $M \approx 3 \times 10^{-7} M_{\odot} \mathrm{yr}^{-1}$ (Rol et al. 2007).

The beaming-corrected energy inferred for GRBs 110709B and $111215 \mathrm{~A}, E_{\gamma}+E_{K} \approx(7-9) \times 10^{51} \mathrm{erg}$, is not unusual for long GRBs, although it is somewhat higher than the median value of $\approx 3 \times 10^{51}$ erg (e.g., Panaitescu \& Kumar 2002; Berger et al. 2003b; Cenko et al. 2011). On the other hand, the inferred densities are substantial, with inferred mass-loss rates of about $6 \times 10^{-5} M_{\odot} \mathrm{yr}^{-1}(110709 \mathrm{~B})$ and $2 \times 10^{-4} M_{\odot} \mathrm{yr}^{-1}(111215 \mathrm{~A})$. At the radii appropriate for the jet break times of the two bursts, 
$r \approx(1.2-1.4) \times 10^{17} \mathrm{~cm}$ (Chevalier \& Li 2000), these mass-loss rates correspond to particle densities of about 100 and $350 \mathrm{~cm}^{-3}$, respectively, higher than typical values for long GRBs, which are $\sim 0.1-30 \mathrm{~cm}^{-3}$ (e.g., Chevalier \& Li 2000; Panaitescu \& Kumar 2002). If confirmed with future detailed observations of dark bursts, the high densities may be indicative of larger mass-loss rates for long GRBs in dusty, and hence metal-rich environments. Such a trend may be expected if the mass loss is driven by radiation pressure mediated by metal lines (Vink \& de Koter 2005).

\subsection{Burst Durations}

The durations of GRB $110709 \mathrm{~B}\left(T_{90} \approx 850 \mathrm{~s}\right)$ and GRB $111215 \mathrm{~A}\left(T_{90} \approx 800 \mathrm{~s}\right)$ are extremely long. To assess whether this type of unusually long duration correlates with optical/near-IR darkness, we collect all Swift bursts with durations of $\gtrsim 500 \mathrm{~s}$, excluding nearby sub-energetic GRBs (e.g., XRF 060218, GRB 100316D) and GRBs without robust $\mathrm{X}$-ray and optical follow-up observations. The sample includes nine events ${ }^{22}$ of which three bursts are dark $(060929,110709 \mathrm{~B}$, 111215A), five have optical and/or near-IR detections with no clear evidence for extinction, and one event lacks rapid followup observations. Thus, at least $\sim 1 / 3$ of these events are dark, similar to the fraction of dark bursts in the overall long GRB population. We therefore conclude that there is no obvious correlation between unusually long duration and darkness, with the caveat that the current sample size is small.

\section{CONCLUSIONS}

Using X-ray, optical/near-IR, and radio observations we have demonstrated that: (1) GRBs 110709B and 111215A are dark bursts; (2) they are robustly associated with galaxies at $z \lesssim 4$ (110709B) and $z \approx 1.8-2.9$ (111215A); (3) they require unusually large rest-frame extinction (among the highest values to date) of $A_{V}^{\text {host }} \gtrsim 5.3 \mathrm{mag}(110709 \mathrm{~B})$ and $\gtrsim 8.5 \mathrm{mag}$ (111215A) at $z=2$; (4) they exhibit commensurately large neutral hydrogen column densities in their $\mathrm{X}$-ray spectra, $N_{\mathrm{H}, \text { int }} \approx(1-3) \times 10^{22} \mathrm{~cm}^{-2}$, which at $z \sim 2$ are consistent with the Galactic $N_{\mathrm{H}}-A_{V}$ relation, unlike the overall long GRB population; and (5) their circumburst environments on a subparsec scale are shaped by large progenitor mass-loss rates of $\approx(6-20) \times 10^{-5} M_{\odot} \mathrm{yr}^{-1}$.

Radio observations played a critical role in this study for three reasons. First, they provided sub-arcsecond positions, which led to a secure identification of the host galaxies. This allowed us to distinguish the extinction scenario from a high-redshift origin. Second, the combination of radio and X-ray data allowed us to robustly determine the required extinction, instead of simply assuming an optical to X-ray spectral index. Indeed, in cases with only X-ray data the unknown location of the synchrotron cooling frequency prevents a unique determination of the extinction. Finally, the radio and X-ray data allowed us to determine the burst parameters, including the geometry, beaming-corrected energy, and the circumburst density. We find that the energy scale for GRBs $110709 \mathrm{~B}$ and $111215 \mathrm{~A}$ is similar to the overall population of optically-bright GRBs. However, the inferred mass-loss rates are larger by about an order magnitude compared with optically-bright bursts, potentially indicating that GRB progenitors in dusty environments have stronger metal line driven winds.

22 These are GRBs 041219A, 050820A, 060123, 060124, 060929, 091024, 110709B, 111016A, and 111215A.
The increased sensitivity of the JVLA played an important role in the study and utilization of the radio afterglow. ALMA will provide similar capabilities, with an expected spatial resolution of about $0 . .04-5^{\prime \prime}$ at $100 \mathrm{GHz}$ (depending on configuration), and hence an assured sub-arcsecond centroiding accuracy for $S / N s$ of $\gtrsim 10$ even in the most compact configuration. As demonstrated here, Chandra observations can also provide subarcsecond positions, but such observations may not be available for all dark GRB candidates (e.g., GRB 111215A). In addition, Chandra data will not enhance the ability to determine the required extinction through broadband modeling.

The JVLA and ALMA will also allow us to study the host galaxies of GRBs 110709B and 111215A (as well as those of past and future dark bursts) in greater detail than optical/nearIR studies alone. In particular, they will address the question of highly-obscured star formation in these galaxies, and may even lead to redshift determinations through the detection of molecular lines. Existing observations with the Very Large Array and the MAMBO and SCUBA bolometers (in the small samples published to-date) have led to only a few detections of GRB hosts (Berger et al. 2003a), with no obvious preference for dark burst hosts (Barnard et al. 2003). However, the enhanced sensitivity of ALMA will allow for detections even with star formation rates of tens of $M_{\odot} \mathrm{yr}^{-1}$ at $z \sim 2$. The combination of detailed host galaxy properties from rest-frame radio to UV, coupled with measurements of the local environments of dark bursts through broadband afterglow modeling will shed light on the location of the obscuring dust (interstellar versus circumstellar) and the impact of metallicity on GRB progenitor formation.

We thank R. Chary for helpful discussions regarding obscured star formation in distant galaxies and Y. Cao for his assistance in attaining the optical spectrum of GRB 111215A while observing at Keck. The Berger GRB group at Harvard is supported by the National Science Foundation under grant AST-1107973, and by NASA/Swift AO7 grant NNX12AD69G. B.A.Z., E.B., R.M., W.F., and A.S. acknowledge partial support of this research while in residence at the Kavli Institute for Theoretical Physics under National Science Foundation Grant PHY1125915. E.N. acknowledges partial support by an ERC starting grant. F.O.E. acknowledges funding of his $\mathrm{PhD}$ through the Deutscher Akademischer Austausch-Dienst (DAAD). A.J.C.T. acknowledges support from the MINECO Spanish Ministry projects AYA 2009-14000-C01 and AYA 2012-39737-C03-01. We thank the referee for useful comments and suggestions.

Funding for GROND was generously granted from the Leibniz Prize to G. Hasinger (DFG grant HA 1850/28-1). D.P. is supported by grant HST-HF-51296.01-A, provided by NASA through a Hubble Fellowship grant from the Space Telescope Science Institute, which is operated by the Association of Universities for Research in Astronomy, Incorporated, under NASA contract NAS5-26555. Support for this work was provided by the National Aeronautics and Space Administration (NASA) through Chandra Award Number 09900712 issued by the Chandra X-ray Observatory Center, which is operated by the SAO for and on behalf of NASA under contract NAS8-03060. The JVLA is operated by the National Radio Astronomy Observatory, a facility of the NSF operated under cooperative agreement by Associated Universities, Inc. JVLA observations were undertaken as part of project numbers 10C-145 and 11B-242. Support for CARMA construction was derived from the Gordon and Betty Moore Foundation, the Kenneth T. and Eileen 
L. Norris Foundation, the James S. McDonnell Foundation, the Associates of the California Institute of Technology, the University of Chicago, the states of California, Illinois, and Maryland, and the NSF. Ongoing CARMA development and operations are supported by the NSF under a cooperative agreement, and by the CARMA partner universities. CARMA observations were undertaken as part of projects c0773 and cx334. The Submillimeter Array is a joint project between the Smithsonian Astrophysical Observatory (SAO) and the Academia Sinica Institute of Astronomy and Astrophysics and is funded by the Smithsonian Institution and the Academia Sinica. SMA observations were undertaken as part of project 2011B-S003. The IRAM Plateau de Bure Interferometer is supported by INSU/CNRS (France), MPG (Germany) and IGN (Spain). Some observations were obtained at the Gemini Observatory, which is operated by the Association of Universities for Research in Astronomy, Inc., under a cooperative agreement with the NSF on behalf of the Gemini partnership: the NSF (United States), the Science and Technology Facilities Council (United Kingdom), the National Research Council (Canada), CONICYT (Chile), the Australian Research Council (Australia), Ministério da Ciência, Tecnologia e Inovação (Brazil), and Ministerio de Ciencia, Tecnología e Innovación Productiva (Argentina). Gemini observations were undertaken as part of programs GS-2011A-Q-25 and GN-2011BQ-10. Some observations were made with the NASA/ESA Hubble Space Telescope, obtained at the Space Telescope Science Institute, which is operated by the Association of Universities for Research in Astronomy, Inc., under NASA contract NAS 5-26555. HST observations were undertaken as part of program 12378. This work utilized observations made with the Gran Telescopio Canarias (GTC), installed in the Spanish Observatorio del Roque de los Muchachos of the Instituto de Astrofsica de Canarias, in the island of La Palma. This research has made use of Swift data obtained from the High Energy Astrophysics Science Archive Research Center (HEASARC), provided by NASA's Goddard Space Flight Center. This research has made use of the XRT Data Analysis Software (XRTDAS) developed under the responsibility of the ASI Science Data Center (ASDC), Italy.

Facilities: Swift (XRT), CXO (ACIS-S), Gemini:South (GMOS), Keck:I (LRIS), HST (ACS, WFC3), GTC, OSN, CARMA, SMA, VLA, IRAM:Interferometer, ESO $2.2 \mathrm{~m} /$ GROND

\section{REFERENCES}

Arnaud, K. A. 1996, in ASP Conf. Ser. 101, Astronomical Data Analysis Software and Systems V, ed. G. H. Jacoby \& J. Barnes (San Francisco, CA: ASP), 17

Barnard, V. E., Blain, A. W., Tanvir, N. R., et al. 2003, MNRAS, 338, 1

Barthelmy, S. D., Barbier, L. M., Cummings, J. R., et al. 2005, SSRv, 120, 143

Barthelmy, S. D., Baumgartner, W. H., Cummings, J. R., et al. 2011a, GCN, 12689,1

Barthelmy, S. D., Burrows, D. N., Cummings, J. R., et al. 2011b, GCN, 12124, 1

Beardmore, A. P., Evans, P. A., Goad, M. R., \& Osborne, J. P. 2011a, GCN, 12136, 1

Beardmore, A. P., Evans, P. A., Goad, M. R., \& Osborne, J. P. 2011b, GCN, 12690, 1

Beckwith, S. V. W., Stiavelli, M., Koekemoer, A. M., et al. 2006, AJ, 132, 1729

Berger, E., Cowie, L. L., Kulkarni, S. R., et al. 2003a, ApJ, 588, 99

Berger, E., Fox, D. B., Kulkarni, S. R., Frail, D. A., \& Djorgovski, S. G. 2007, ApJ, 660, 504

Berger, E., Kulkarni, S. R., Bloom, J. S., et al. 2002, ApJ, 581, 981

Berger, E., Kulkarni, S. R., Pooley, G., et al. 2003b, Natur, 426, 154

Bouwens, R. J., Illingworth, G. D., Franx, M., et al. 2009, ApJ, 705, 936

Burrows, D. N., Hill, J. E., Nousek, J. A., et al. 2005, SSRv, 120, 165

Campana, S., Romano, P., Covino, S., et al. 2006, A\&A, 449, 61
Campana, S., Salvaterra, R., Melandri, A., et al. 2012, MNRAS, 421, 1697

Castro-Tirado, A. J., Bremer, M., McBreen, S., et al. 2007, A\&A, 475, 101

Cenko, S. B., Frail, D. A., Harrison, F. A., et al. 2011, ApJ, 732, 29

Cenko, S. B., Kelemen, J., Harrison, F. A., et al. 2009, ApJ, 693, 1484

Chevalier, R. A., \& Li, Z.-Y. 2000, ApJ, 536, 195

Cucchiara, A., Levan, A. J., Fox, D. B., et al. 2011, ApJ, 736, 7

Cummings, J. R., Barthelmy, S. D., Baumgartner, W. H., et al. 2011a, GCN, 12144,1

Cummings, J. R., Barthelmy, S. D., Burrows, D. N., et al. 2011b, GCN, 12122,

D’Avanzo, A., Melandri, A., Covino, S., et al. 2011, GCN, 12695, 1 de Ugarte Postigo, A., Lundgren, A., De Breuck, C., et al. 2011, GCN, 12151,

1

Djorgovski, S. G., Frail, D. A., Kulkarni, S. R., et al. 2001, ApJ, 562, 654

Djorgovski, S. G., Kulkarni, S. R., Bloom, J. S., et al. 1998, ApJL, 508, L17

Fruchter, A. S., \& Hook, R. N. 2002, PASP, 114, 144

Fruchter, A. S., Levan, A. J., Strolger, L., et al. 2006, Natur, 441, 463

Fynbo, J. P. U., Jakobsson, P., Prochaska, J. X., et al. 2009, ApJS, 185, 526

Fynbo, J. U., Jensen, B. L., Gorosabel, J., et al. 2001, A\&A, 369, 373

Galama, T. J., \& Wijers, R. A. M. J. 2001, ApJL, 549, L209

Golenetskii, S., Aptekar, R., Frederiks, D., et al. 2011, GCN, 12135, 1

Gorbovskoy, E., Lipunov, V., Kornilov, V., et al. 2011, GCN, 12687, 1

Granot, J., \& Sari, R. 2002, ApJ, 568, 820

Greiner, J., Bornemann, W., Clemens, C., et al. 2007, Msngr, 130, 12

Greiner, J., Bornemann, W., Clemens, C., et al. 2008, PASP, 120, 405

Greiner, J., Krühler, T., Klose, S., et al. 2011, A\&A, 526, A30

Greisen, E. W. 2003, in Information Handling in Astronomy-Historical Vistas, ed. A. Heck (Astrophysics and Space Science Library, Vol. 285; Dordrecht: Kluwer), 109

Groot, P. J., Galama, T. J., van Paradijs, J., et al. 1998, ApJL, 493, L27

Güver, T., \& Özel, F. 2009, MNRAS, 400, 2050

Haislip, J. B., Nysewander, M. C., Reichart, D. E., et al. 2006, Natur, 440, 181

Ho, P. T. P., Moran, J. M., \& Lo, K. Y. 2004, ApJL, 616, L1

Hogg, D. W., Pahre, M. A., McCarthy, J. K., et al. 1997, MNRAS, 288, 404

Holland, S. T., \& Cummings, J. R. 2011, GCN, 12157, 1

Holland, S. T., Sbarufatti, B., Shen, R., et al. 2010, ApJ, 717, 223

Hook, I. M., Jørgensen, I., Allington-Smith, J. R., et al. 2004, PASP, 116, 425

Jakobsson, P., Frail, D. A., Fox, D. B., et al. 2005, ApJ, 629, 45

Jakobsson, P., Fynbo, J. P. U., Ledoux, C., et al. 2006, A\&A, 460, L13

Jakobsson, P., Hjorth, J., Fynbo, J. P. U., et al. 2004, ApJL, 617, L21

Jaunsen, A. O., Rol, E., Watson, D. J., et al. 2008, ApJ, 681, 453

Kalberla, P. M. W., Burton, W. B., Hartmann, D., et al. 2005, A\&A, 440, 775

Kann, D. A., Klose, S., \& Zeh, A. 2006, ApJ, 641, 993

Kann, D. A., Klose, S., Zhang, B., et al. 2010, ApJ, 720, 1513

Kennicutt, R. C., Jr. 1998, ARA\&A, 36, 189

Kimble, R. A., MacKenty, J. W., O'Connell, R. W., \& Townsend, J. A. 2008, Proc. SPIE, 7010, 43

Koekemoer, A. M., Fruchter, A. S., Hook, R. N., \& Hack, W. 2002, in The 2002 HST Calibration Workshop: Hubble After the ACS and the NICMOS Cooling System, ed. S. Arribas, A. Koekemoer, \& B. Whitmore (Baltimore, MD: Space Telescope Science Institute), 337

Krühler, T., Greiner, J., Schady, P., et al. 2011, A\&A, 534, A108

Krühler, T., Küpcü Yoldaş, A., Greiner, J., et al. 2008, ApJ, 685, 376

Levan, A., Fruchter, A., Rhoads, J., et al. 2006, ApJ, 647, 471

Levesque, E. M., Kewley, L. J., Graham, J. F., \& Fruchter, A. S. 2010, ApJL, 712, L26

Margutti, R., Genet, F., Granot, J., et al. 2010, MNRAS, 402, 46

Margutti, R., Zaninoni, E., Bernardini, M. G., et al. 2013, MNRAS, 428, 729

Melandri, A., Mundell, C. G., Kobayashi, S., et al. 2008, ApJ, 686, 1209

Melandri, A., Sbarufatti, B., D’Avanzo, P., et al. 2012, MNRAS, 421, 1265

Murphy, E. J., Chary, R.-R., Dickinson, M., et al. 2011, ApJ, 732, 126

Oates, S. R. 2011, GCN, 12693, 1

Oates, S. R., Barthelmy, S. D., Baumgartner, W. H., et al. 2011, GCN, 12681, 1

Oates, S. R., Barthelmy, S. D., Beardmore, A., et al. 2012, GCNR, 358, 1

Oke, J. B., Cohen, J. G., Carr, M., et al. 1995, PASP, 107, 375

Panaitescu, A., \& Kumar, P. 2002, ApJ, 571, 779

Pandey, S. B., Roy, R., Kumar, B., \& Yadav, R. K. S. 2011, GCN, 12686, 1

Perley, D. A., Cenko, S. B., Bloom, J. S., et al. 2009, AJ, 138, 1690

Perley, D. A., Levan, A. J., Tanvir, N. R., et al. 2013, ApJ, submitted (arXiv:1301.5903)

Perley, D. A., Morgan, A. N., Updike, A., et al. 2011a, AJ, 141, 36

Perley, R. A., Chandler, C. J., Butler, B. J., \& Wrobel, J. M. 2011b, ApJL, 739, L1

Piro, L., Frail, D. A., Gorosabel, J., et al. 2002, ApJ, 577, 680

Predehl, P., \& Schmitt, J. H. M. M. 1995, A\&A, 293, 889

Prochaska, J. X., Sheffer, Y., Perley, D. A., et al. 2009, ApJL, 691, L27

Ramirez-Ruiz, E., Trentham, N., \& Blain, A. W. 2002, MNRAS, 329, 465 
Reddy, N. A., \& Steidel, C. C. 2009, ApJ, 692, 778

Reichart, D. E., \& Price, P. A. 2002, ApJ, 565, 174

Rockosi, C., Stover, R., Kibrick, R., et al. 2010, Proc. SPIE, 7735, 26

Rol, E., van der Horst, A., Wiersema, K., et al. 2007, ApJ, 669, 1098

Roming, P. W. A., Kennedy, T. E., Mason, K. O., et al. 2005, SSRv, 120, 95

Roseboom, I. G., Bunker, A., Sumiyoshi, M., et al. 2012, MNRAS, 426, 1782

Rossi, A., Klose, S., Ferrero, P., et al. 2012, A\&A, 545, A77

Rumyantsev, V., Pit', N., Volnova, A., et al. 2011, GCN, 12703, 1

Salvaterra, R., Della Valle, M., Campana, S., et al. 2009, Natur, 461, 1258

Sánchez, B., Aguiar-González, M., Barreto, R., et al. 2012, Proc. SPIE, 8446, 4

Sari, R., Piran, T., \& Halpern, J. P. 1999, ApJL, 519, L17

Sari, R., Piran, T., \& Narayan, R. 1998, ApJL, 497, L17

Sault, R. J., Teuben, P. J., \& Wright, M. C. H. 1995, in ASP Conf. Ser. 77, Astronomical Data Analysis Software and Systems IV, ed. R. A. Shaw, H. E. Payne, \& J. J. E. Hayes (San Francisco, CA: ASP), 433

Schady, P., Mason, K. O., Page, M. J., et al. 2007, MNRAS, 377, 273

Schlafly, E. F., \& Finkbeiner, D. P. 2011, ApJ, 737, 103

Siringo, G., Kreysa, E., Kovács, A., et al. 2009, A\&A, 497, 945

Stratta, G., Fiore, F., Antonelli, L. A., Piro, L., \& De Pasquale, M. 2004, ApJ, 608,846
Svensson, K. M., Levan, A. J., Tanvir, N. R., et al. 2012, MNRAS, 421, 25

Tanvir, N. R., Barnard, V. E., Blain, A. W., et al. 2004, MNRAS, 352, 1073

Tanvir, N. R., Fox, D. B., Levan, A. J., et al. 2009, Natur, 461, 1254

Tanvir, N. R., Wiersema, K., Melandri, A., et al. 2011, GCN, 12696, 1

Trentham, N., Ramirez-Ruiz, E., \& Blain, A. W. 2002, MNRAS, 334, 983

Usui, R., Aoki, Y., Song, S., et al. 2011, GCN, 12685, 1

van der Horst, A. J., Kouveliotou, C., Gehrels, N., et al. 2009, ApJ, 699, 1087

Vink, J. S., \& de Koter, A. 2005, A\&A, 442, 587

Wainwright, C., Berger, E., \& Penprase, B. E. 2007, ApJ, 657, 367

Watson, D. 2011, A\&A, 533, A16

Watson, D., Fynbo, J. P. U., Ledoux, C., et al. 2006, ApJ, 652, 1011

Watson, D., \& Jakobsson, P. 2012, ApJ, 754, 89

Woosley, S. E., \& Bloom, J. S. 2006, ARA\&A, 44, 507

Wright, E. L. 2006, PASP, 118, 1711

Xin, L. P., Wei, J. Y., Qiu, Y. L., et al. 2011, GCN, 12682,

Xu, D., Zhao, X.-H., Mao, J.-R., \& Bai, J.-M. 2011, GCN, 12683, 1

Zafar, T., Watson, D., Fynbo, J. P. U., et al. 2011, A\&A, 532, A143

Zhang, B.-B., Burrows, D. N., Zhang, B., et al. 2012, ApJ, 748, 132 